\title{
Regioselective Synthesis of Benzofuranones and Benzofurans
}

\author{
Xiaojie Zhang and Christopher M. Beaudry* \\ Department of Chemistry, Oregon State University, 153 Gilbert Hall, Corvallis, OR \\ 97331, United States \\ christopher.beaudry@oregonstate.edu
}

\section{Table of Contents}

General Experimental Details S002

Experimental Procedures S003

Depicted ${ }^{1} \mathrm{H}$ and ${ }^{13} \mathrm{C}$ NMR Spectra S006 


\section{General Experimental Details:}

All reactions were carried out under an inert Ar atmosphere in oven-dried glassware. External (heated oil or cryogenic solvent) bath temperatures were used to record all reaction temperatures. Flash column chromatography (FCC) was carried out with SiliaFlash P60, 60 A silica gel. Reactions and column chromatography were monitored with EMD silica gel 60 F254 plates and visualized with potassium permanganate stain. Reagent grade 1,4-dioxane was dried over $\mathrm{CaH}_{2}$ and distilled prior to use. 1,2Dichlorobenzene (DCB) was distilled under reduced pressure and degassed using three freeze-pump-thaw cycles. Tetrahydrofuran (THF) and methylene chloride $\left(\mathrm{CH}_{2} \mathrm{Cl}_{2}\right)$ were dried by passage through activated alumina columns. All other reagents and solvents were used without further purification from commercial sources. Unless otherwise noted, melting points were obtained from material that solidified after chromatography.

Instrumentation: FT-IR spectra were obtained on $\mathrm{NaCl}$ plates with a PerkinElmer Spectrum Vision spectrometer. HRMS were recorded on a JEOL MS Route Magnetic Sector Instrument (EI) or a Waters Synapt HDMS TOF instrument (ESI). Proton and carbon NMR spectra $\left({ }^{1} \mathrm{H}\right.$ NMR and ${ }^{13} \mathrm{C}$ NMR $)$ were recorded in deuterated chloroform (CDCl3) unless otherwise noted on a Bruker $700 \mathrm{MHz}$ Avance III Spectrometer with carbon-optimized cryoprobe or Bruker $400 \mathrm{MHz}$ DPX-400 spectrometer. Multiplicities are abbreviated as follows: $\mathrm{s}=$ singlet, $\mathrm{d}=$ doublet, $\mathrm{t}=$ triplet, $\mathrm{q}=$ quartet, $\mathrm{br}=$ broad, $\mathrm{m}=$ multiplet. Melting points were determined with a Cole-Parmer instrument and are uncorrected. 


\section{Experimental Procedures:}

\section{Preparation of Nitroalkene Coupling Partners}

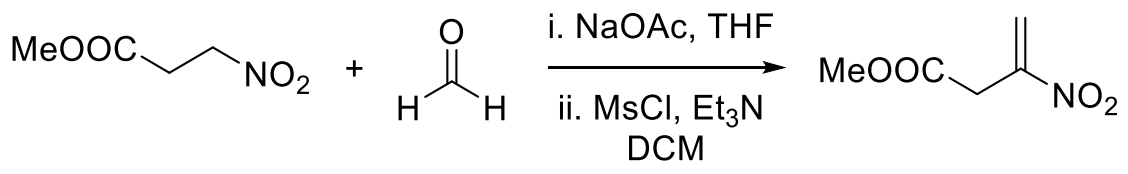

\section{Methyl 3-nitrobut-3-enoate (18-S1)}

To a solution of methyl 3-nitropropanoate $(0.910 \mathrm{~g}, 7.00 \mathrm{mmol}, 1.0$ equiv) and paraformaldehyde ( $1.05 \mathrm{~g}, 35.0 \mathrm{mmol}, 5.0$ equiv) in THF $(35.0 \mathrm{ml})$ was added $\mathrm{NaOAc}$ $\left(0.170 \mathrm{~g}, 2.10 \mathrm{mmol}, 0.30\right.$ equiv) at $0{ }^{\circ} \mathrm{C}$. The mixture was stirred at $0{ }^{\circ} \mathrm{C}$ for $30 \mathrm{mins}$ and then warmed to $\mathrm{rt}$ for 2 days. The mixture was filtered through Celite and eluted with EtOAc. The filtrate was concentrated, and the residue was purified by FCC (3:1 hexanes:EtOAc) to yield an alcohol product as an oil (868 $\mathrm{mg}, 76 \%)$

To a solution of the corresponding alcohol (1.06 g, $6.50 \mathrm{mmol}, 1.0$ equiv) in $\mathrm{CH}_{2} \mathrm{Cl}_{2}$ $(22.0 \mathrm{ml}, 0.3 \mathrm{M})$ at $0{ }^{\circ} \mathrm{C}$ was added $\mathrm{MsCl}(1.11 \mathrm{~g}, 9.75 \mathrm{mmol}, 1.5$ equiv). The mixture was stirred at $0{ }^{\circ} \mathrm{C}$ for $30 \mathrm{~min}$, upon which time $\mathrm{Et}_{3} \mathrm{~N}$ (1.31 g, $13.0 \mathrm{mmol}, 2.0$ equiv) was added dropwise over $30 \mathrm{~min}$. Following the addition of $\mathrm{Et}_{3} \mathrm{~N}$, the mixture was quenched with $1 \mathrm{~N} \mathrm{HCl}(50 \mathrm{ml})$, and extracted with EtOAc $(5 \times 30 \mathrm{~mL})$. The combined organic layers were dried over $\mathrm{Na}_{2} \mathrm{SO}_{4}$, filtered, and concentrated. The residue was purified by FCC (5:1 hexanes:EtOAc) to yield 18-S1 as an oil (869 mg, 92\%).

Data for 18-S1: Rf: 0.48 (3:2 hexanes:EtOAc); IR: (thin film) 2957, 1741, 1530, 1346 $\mathrm{cm}^{-1} ;{ }^{1} \mathrm{H}$ NMR $\left(700 \mathrm{MHz}, \mathrm{CDCl}_{3}\right) \delta 6.66(\mathrm{~d}, J=2.1 \mathrm{~Hz}, 1 \mathrm{H}), 5.81(\mathrm{~s}, 1 \mathrm{H}), 3.74(\mathrm{~s}, 3$ $\mathrm{H}), 3.65(\mathrm{~s}, 2 \mathrm{H}) ;{ }^{13} \mathrm{C}\left\{{ }^{1} \mathrm{H}\right\} \mathrm{NMR}\left(176 \mathrm{MHz}, \mathrm{CDCl}_{3}\right) \delta 168.5,151.0,121.5,52.7,36.1$; HRMS (ESI) m/z: [M+ H] ${ }^{+}$Calcd for $\mathrm{C}_{5} \mathrm{H}_{8} \mathrm{NO}_{4}$ 146.0453; Found 146.0454.

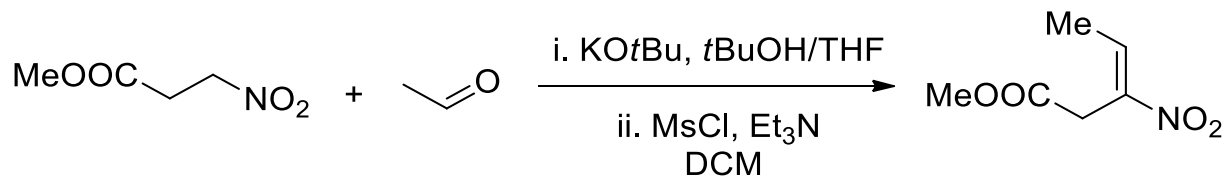

\section{Methyl-3-nitropent-3-enoate (18-S2)}

To a solution of methyl 3-nitropropanoate (1.29 g, $9.70 \mathrm{mmol}, 1.0$ equiv) in $t \mathrm{BuOH} / \mathrm{THF}(1: 1)(19.4 \mathrm{ml}, 0.5 \mathrm{M})$ at $0{ }^{\circ} \mathrm{C}$ was added $\mathrm{KO} t \mathrm{Bu}(0.109 \mathrm{~g}, 0.970 \mathrm{mmol}$, 0.10 equiv). The mixture was stirred at $0{ }^{\circ} \mathrm{C}$ for $10 \mathrm{mins}$, upon which time acetaldehyde ( $0.850 \mathrm{~g}, 19.4 \mathrm{mmol}, 2.0$ equiv) was added dropwise. The mixture was stirred at $0{ }^{\circ} \mathrm{C}$ for $10 \mathrm{~min}$ and then warmed to rt. The mixture was stirred at $\mathrm{rt}$ for an additional $16 \mathrm{~h}$. The mixture was quenched with $1 \mathrm{~N} \mathrm{HCl}(50 \mathrm{ml})$, and extracted with EtOAc $(5 \times 30$ $\mathrm{ml})$. The combined organic layers were dried over $\mathrm{Na}_{2} \mathrm{SO}_{4}$, filtered and concentrated. To a solution of the crude material $\left(1.72 \mathrm{~g}, 9.70 \mathrm{mmol}, 1.0\right.$ equiv) from above in $\mathrm{CH}_{2} \mathrm{Cl}_{2}$ (33.0 mL, $0.3 \mathrm{M}$ ) at $0{ }^{\circ} \mathrm{C}$ was added $\mathrm{MsCl}(1.66 \mathrm{~g}, 14.6 \mathrm{mmol}, 1.5$ equiv). The mixture was stirred at $0{ }^{\circ} \mathrm{C}$ for $15 \mathrm{~min}$, upon which time Et $3 \mathrm{~N}$ ( $2.94 \mathrm{~g}, 29.1 \mathrm{mmol}, 3.0$ equiv) was added dropwise over $20 \mathrm{~min}$. The mixture was stirred at $0{ }^{\circ} \mathrm{C}$ for $10 \mathrm{~min}$. The 
mixture was quenched with $1 \mathrm{~N} \mathrm{HCl}(30 \mathrm{~mL})$, and extracted with EtOAc $(3 \times 30 \mathrm{~mL})$. The combined organic layers were dried over $\mathrm{Na}_{2} \mathrm{SO}_{4}$, filtered, and concentrated. The residue was purified by FCC (10:1 hexanes:EtOAc) to yield 18-S2 as an oil (597 $\mathbf{m g}$, $39 \%)$.

Data for 18-S2: Rf: 0.5 (3:1 hexanes:EtOAc); IR: (thin film) 2956, 2849, 1742, 1521, $1338 \mathrm{~cm}^{-1}$; ${ }^{1} \mathrm{H}$ NMR $\left(700 \mathrm{MHz}, \mathrm{CDCl}_{3}\right) \delta 7.45$ (q, $\left.J=7.7 \mathrm{~Hz}, 1 \mathrm{H}\right), 3.74(\mathrm{~s}, 3 \mathrm{H}), 3.65$ $(\mathrm{s}, 2 \mathrm{H}), 1.95(\mathrm{~d}, J=7.7 \mathrm{~Hz}, 3 \mathrm{H}) ;{ }^{13} \mathrm{C}\left\{{ }^{1} \mathrm{H}\right\} \mathrm{NMR}\left(176 \mathrm{MHz}, \mathrm{CDCl}_{3}\right) \delta 168.8,145.8$, 135.5, 52.6, 31.9, 14.0; HRMS (ESI) m/z: $[\mathrm{M}+\mathrm{H}]^{+}$Calcd for $\mathrm{C}_{6} \mathrm{H}_{10} \mathrm{NO}_{4}$ 160.0610; Found 160.0616.

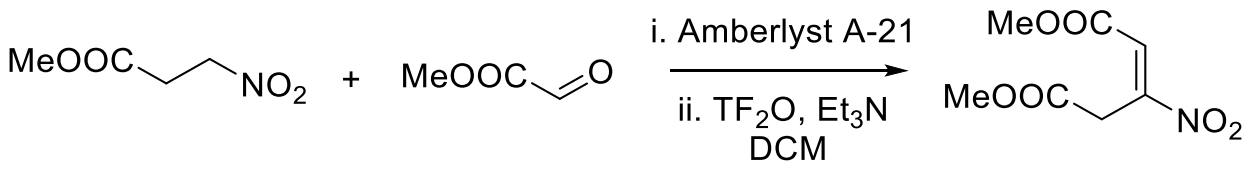

To methyl 3-nitropropanoate $\left(0.950 \mathrm{~g}, 7.18 \mathrm{mmol}, 1.0\right.$ equiv) at $0{ }^{\circ} \mathrm{C}$ was added amberlyst A-21 $(0.540 \mathrm{~g})$. The mixture was stirred at $0{ }^{\circ} \mathrm{C}$ for $5 \mathrm{~min}$, upon which time methyl glyoxalate $(0.950 \mathrm{~g}, 10.7 \mathrm{mmol}, 0.50$ equiv) was added. The mixture was stirred at $0{ }^{\circ} \mathrm{C}$ for $30 \mathrm{~min}$ and then warmed to $\mathrm{rt}$ for 2 days. The mixture was filtered through Celite with EtOAc to remove solids. The filtrate was concentrated and crude material was used in the next step without additional purification.

To a solution of the crude material ( $1.48 \mathrm{~g}, 7.18 \mathrm{mmol}, 1.0$ equiv) from above in $\mathrm{CH}_{2} \mathrm{Cl}_{2}$ $(36.0 \mathrm{~mL}, 0.2 \mathrm{M})$ at $0{ }^{\circ} \mathrm{C}$ was added $\mathrm{Tf}_{2} \mathrm{O}(2.40 \mathrm{~g}, 8.60 \mathrm{mmol}, 1.2$ equiv). The mixture was stirred at $0{ }^{\circ} \mathrm{C}$ for $10 \mathrm{~min}$, and then Et $3 \mathrm{~N}$ (1.45 g, $14.4 \mathrm{mmol}, 2.0$ equiv) was added dropwise. The mixture was stirred at $0{ }^{\circ} \mathrm{C}$ for $2 \mathrm{~h}$. The mixture was quenched with $1 \mathrm{~N}$ $\mathrm{HCl}(30 \mathrm{~mL})$, and extracted with EtOAc $(3 \times 30 \mathrm{~mL})$. The combined organic layers were dried over $\mathrm{Na}_{2} \mathrm{SO}_{4}$, filtered, and concentrated. The residue was purified by FCC (10:1 hexanes:EtOAc) to yield 18-S3 as an oil (462 mg, 32\%).

Data for 18-S3: Rf: 0.59 (3:2 hexanes:EtOAc); IR: (thin film) 2958, 1729, 1541, 1437, $1323 \mathrm{~cm}^{-1} ;{ }^{1} \mathrm{H}$ NMR $\left(700 \mathrm{MHz}, \mathrm{CDCl}_{3}\right) \delta 7.28(\mathrm{~s}, 1 \mathrm{H}), 4.25(\mathrm{~s}, 2 \mathrm{H}), 3.86(\mathrm{~s}, 3 \mathrm{H})$, $3.74(\mathrm{~s}, 3 \mathrm{H}) ;{ }^{13} \mathrm{C}\left\{{ }^{1} \mathrm{H}\right\} \mathrm{NMR}\left(176 \mathrm{MHz}, \mathrm{CDCl}_{3}\right) \delta 167.9,164.1,156.1,123.8,53.0$, 52.8, 32.8; HRMS (ESI) m/z: $[\mathrm{M}+\mathrm{H}]^{+}$Calcd for $\mathrm{C}_{7} \mathrm{H}_{10} \mathrm{NO}_{6} 204.0508$; Found 204.0504. 


\section{Converting Phenol (23) to Benzofuranone (24)}<smiles>COC(=O)Cc1ccccc1O</smiles>

To a thick-walled reaction vessel were added phenol 23 (16.6 mg, $0.100 \mathrm{mmol}, 1.0$ equiv), BHT (2.20 mg, $0.0100 \mathrm{mmol}, 0.10$ equiv) and $\mathrm{AlCl}_{3}(1.33 \mathrm{mg}, 0.0100 \mathrm{mmol}$, 0.10 equiv). The vessel was flushed with Ar gas for $5 \mathrm{~min}$. DCB $(0.330 \mathrm{ml}, 0.3 \mathrm{M})$ and TFA (2.28 mg, $0.0200 \mathrm{mmol}, 0.20$ equiv) were added, and tube was quickly sealed. The reaction mixture was heated to $150{ }^{\circ} \mathrm{C}$ for $4 \mathrm{~h}$. Using $\mathrm{CH}_{2} \mathrm{Br}_{2}(1.74 \mathrm{mg}, 0.100 \mathrm{mmol})$ as internal standard, NMR showed benzofuranone $24(0.070 \mathrm{mmol}, 70 \%)$ and phenol $23(0.010 \mathrm{mmol}, 10 \%)$ in the reaction mixture. 


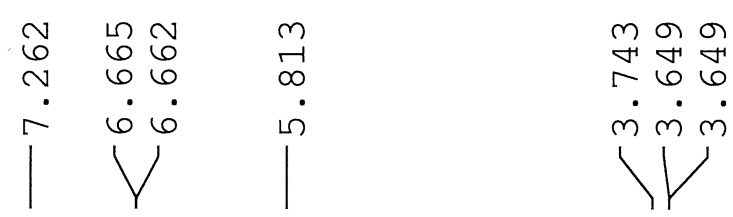

MeOOC $\sim_{\mathrm{NO}_{2}}$

18-S1

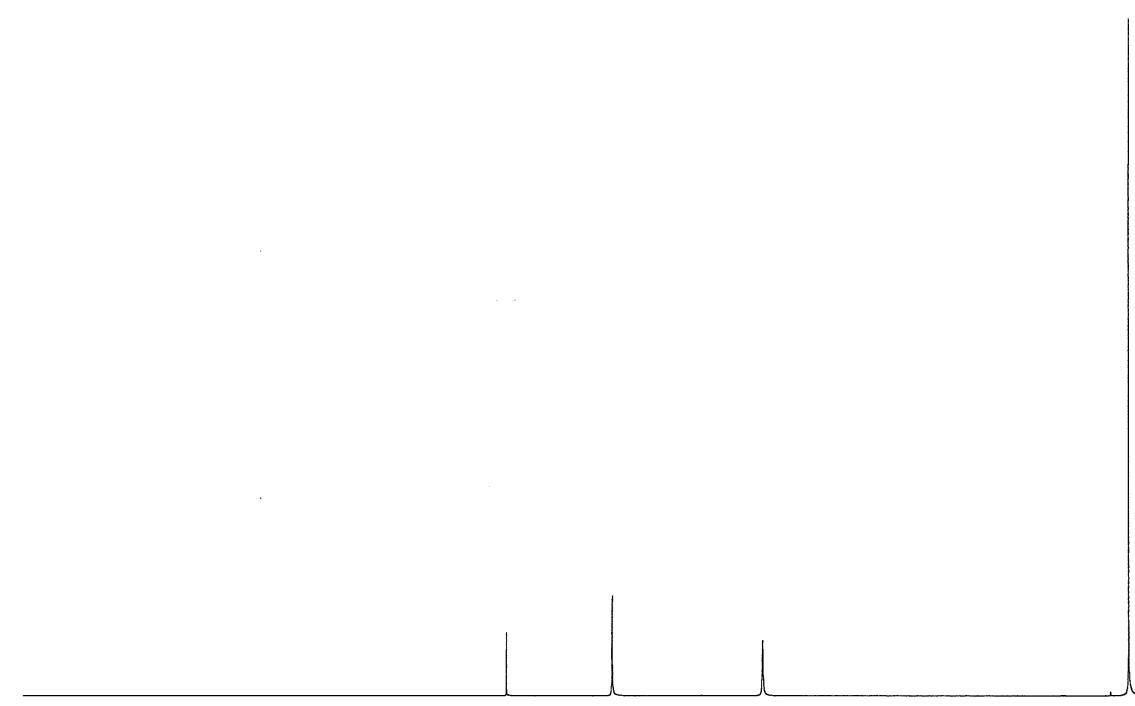

9

8

7

6

(1)
54

के

\section{BRONER $C_{-\infty}$}

Current Data Parameters

NAME

EXPNO

$\mathrm{XZ}-4-140$

F2 - Acquisition Parameter

Time

INSTRUM

PROB HD

PULPROG

TD

SOLVENT

NS

DS

FIDRE

$\mathrm{AQ}$
$\mathrm{RG}$

RG

DE

TE

TD 0

$\mathrm{SFO1}$

NOC1

PO

P LW1

20200109

$14.44 \mathrm{~h}$

z151340 0001

_0001

65536

CDCl3

16

$10504.202 \mathrm{~Hz}$

$0.320563 \mathrm{~Hz}$

$3.1195135 \mathrm{sec}$

36

47.600 usec

20.00 usec

$303.2 \mathrm{~K}$

$1.00000000 \mathrm{sec}$

$700.0115500 \mathrm{MHz}$ $1 \mathrm{H}$

3.17 usec

3.17 usec
9.50 usec

$13.00000000 \mathrm{~W}$

F2 - Processing parameters

SI $\quad 131072$

$\begin{array}{lc}\text { SF } & 700.0070169 \mathrm{MHz} \\ \text { WDW } & \mathrm{EM}\end{array}$

SSB

$\mathrm{LB}$

GB
PC

0

EM

$0.30 \mathrm{~Hz}$

1.00 


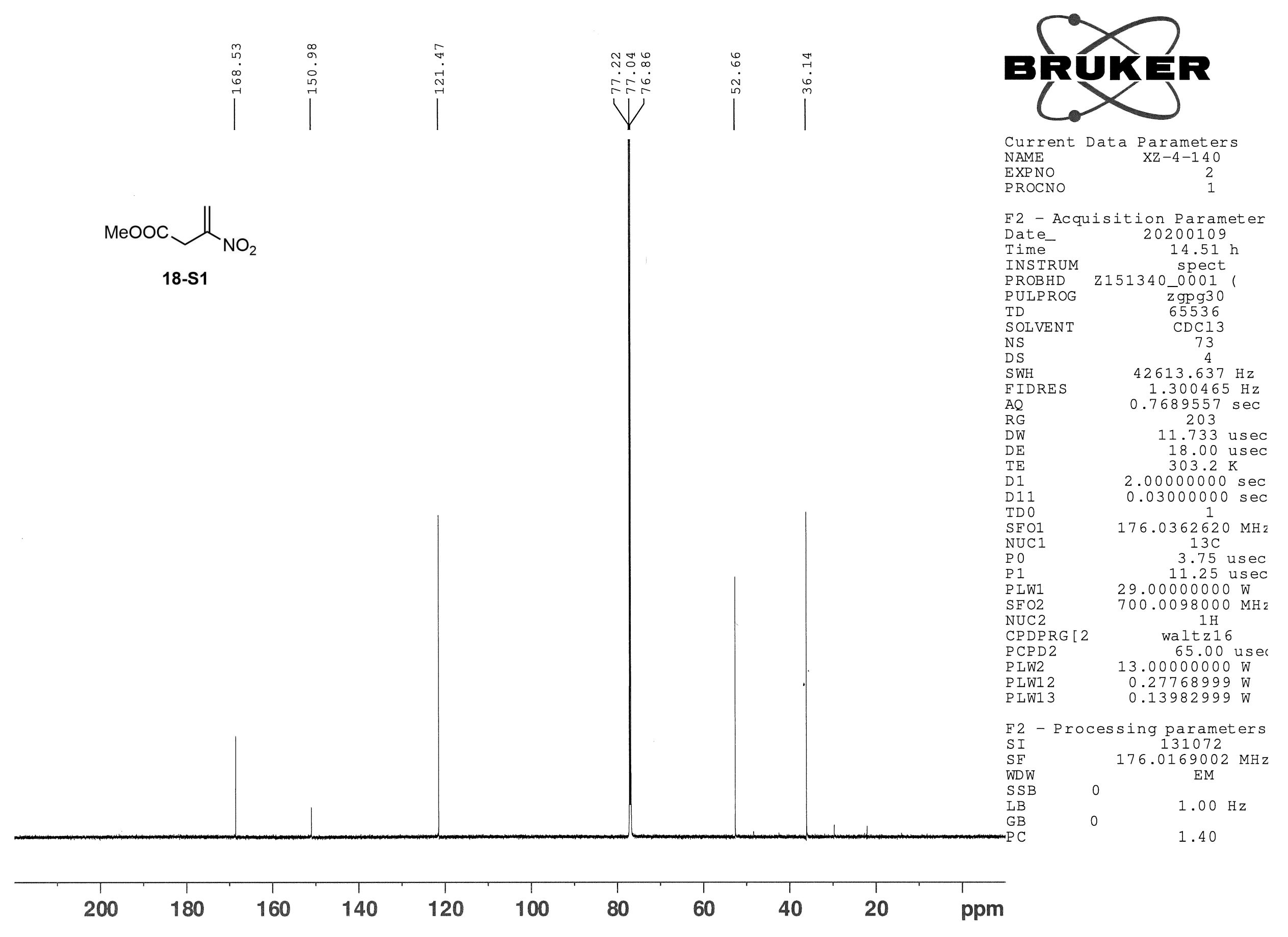




$A$ A

$\therefore \sim r \dot{r}$

4

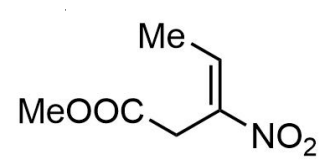

18-S2

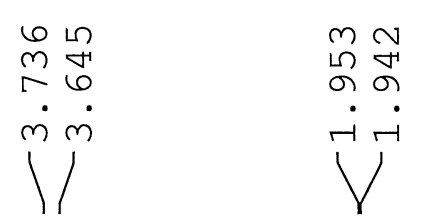

ฝั ๙ั

각

BiUMER $C$

Current Data Parameters NAME EXPNO PROCNO $\mathrm{XZ}-4-229$

F2 - Acquisition Parameter Date_ 20200626 Time INSTRUM

PROBHD

P ULPROG $18.26 \mathrm{~h}$

2151340_0001

TD

SOLVENT

NS

SWH

FIDRES

$A Q$

RG

DW

DE

TE

D 1

TDO

SFO1

NUC

P 0

P IW1

zg30

65536

$\mathrm{CDCl} 3$ 16
2

$10504.202 \mathrm{~Hz}$ $0.320563 \mathrm{~Hz}$ $3.1195135 \mathrm{sec}$ 40.3

47.600 usec 20.00 usec $303.2 \mathrm{~K}$

$1.00000000 \mathrm{sec}$ $700.0115500 \mathrm{MHz}$ $1 \mathrm{H}$
3.17 usec
9.50 usec

13.00000000

F2 - Processing parameters SI $\quad 131072$

SF $\quad 700.0070163 \mathrm{MHz}$

EM

S SB

LB

0

$0.30 \mathrm{~Hz}$

$\mathrm{PC}$

1.00

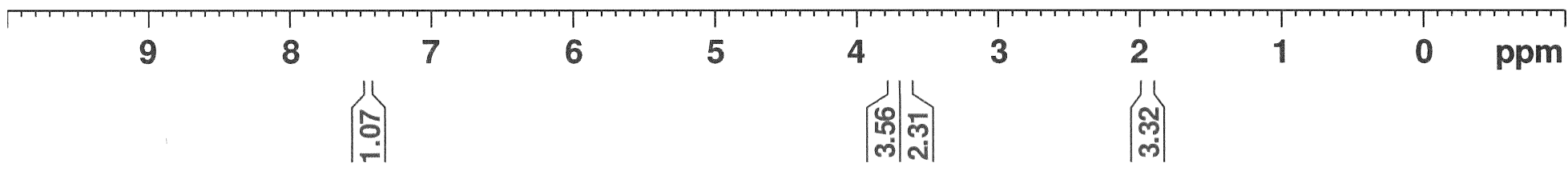




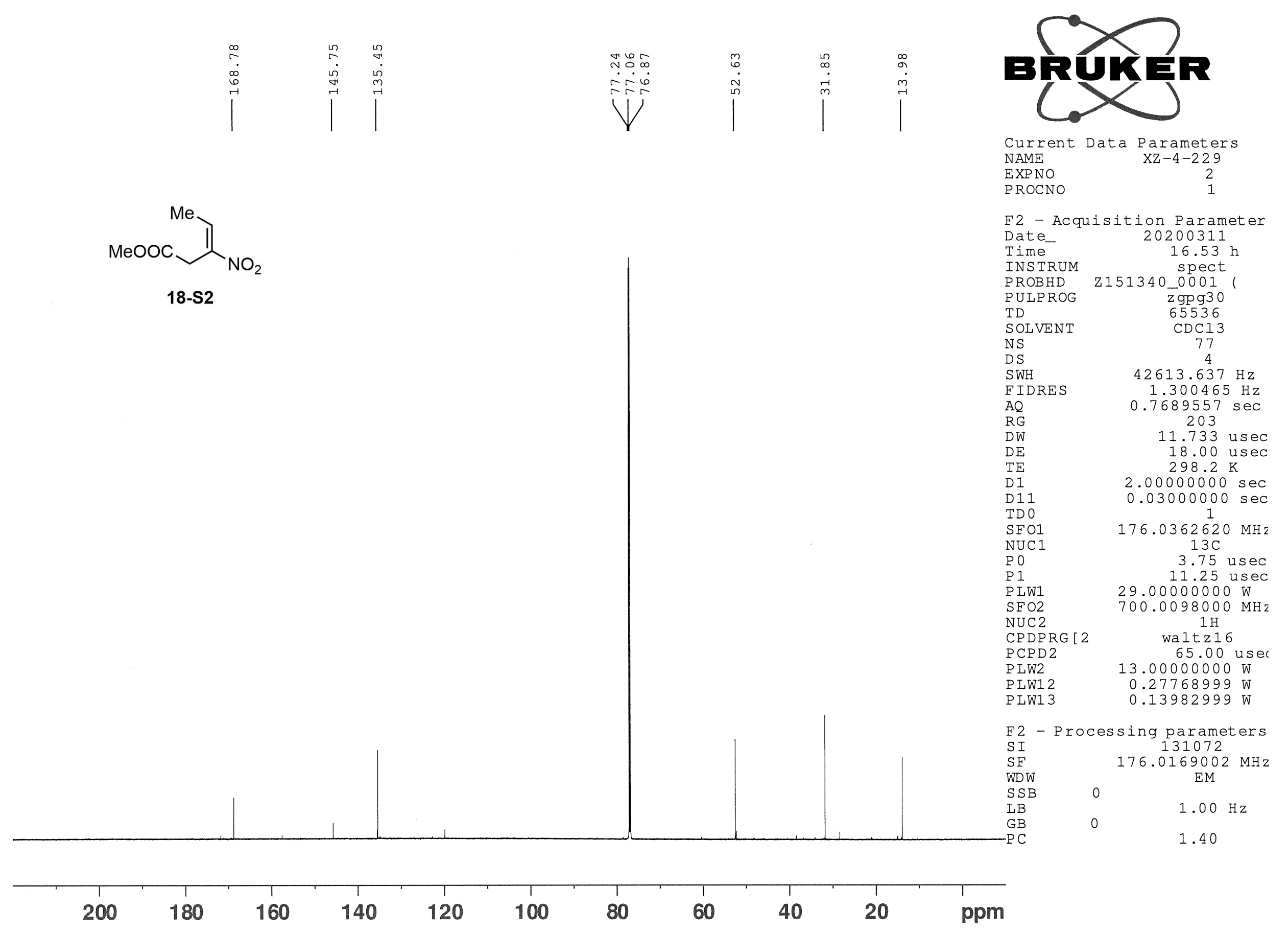




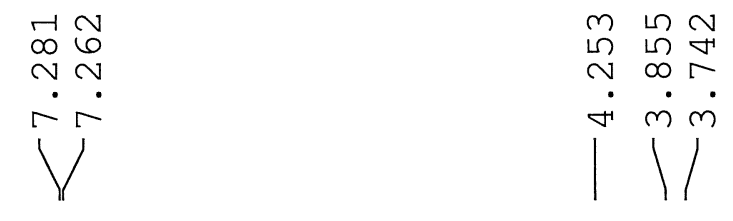

MeOOC $\sim_{\mathrm{NO}_{2}}^{\mathrm{MeOOC}}$

18-S3

\section{BR}

Current Data Parameters

NAME

EXPNO

F2 - Acquisition Parameter

Time

INSTRUM

PROBHD

PULPROG

10.35

$10.35 \mathrm{~h}$

TD

z151340_0001

SOLVENT

NS

DS

FIDRES

FIDRES

$\mathrm{AQ}$

RG

DW

DE

TE

D 1

SFO1

$\mathrm{SFO1}$

P 0

P 1
P LW1

$\mathrm{CDCl} 3$ 16

$10504.202 \mathrm{~Hz}$ $0.320563 \mathrm{~Hz}$ $3.1195135 \mathrm{sec}$ 36

47.600 usec 20.00 usec $298.2 \mathrm{~K}$

$1.00000000 \mathrm{sec}$ $700.0115500 \mathrm{MHz}$ 3.17 usec 9.50 usec

2 Processing parameters

SI 131072

SF $\quad 700.0070148 \mathrm{MHz}$

EM

S SB

$\mathrm{LB}$

0

$0.30 \mathrm{~Hz}$

$\mathrm{GB}$
$\mathrm{PC}$

1.00

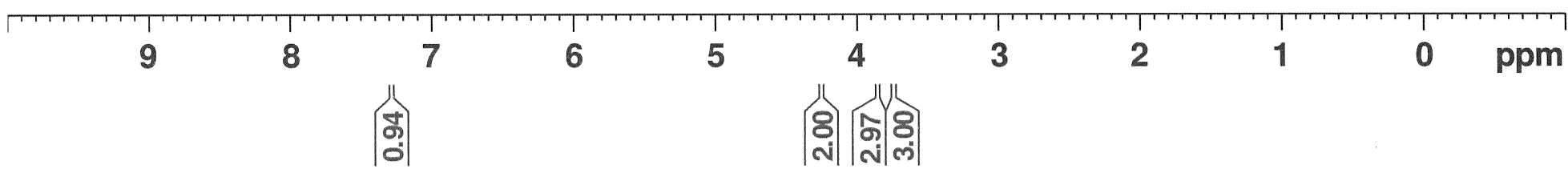




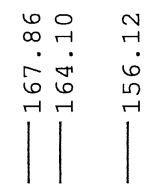

$\mathrm{MeOOC} \sim_{\mathrm{NO}_{2}}^{\mathrm{MeOOC}}$

18-S3
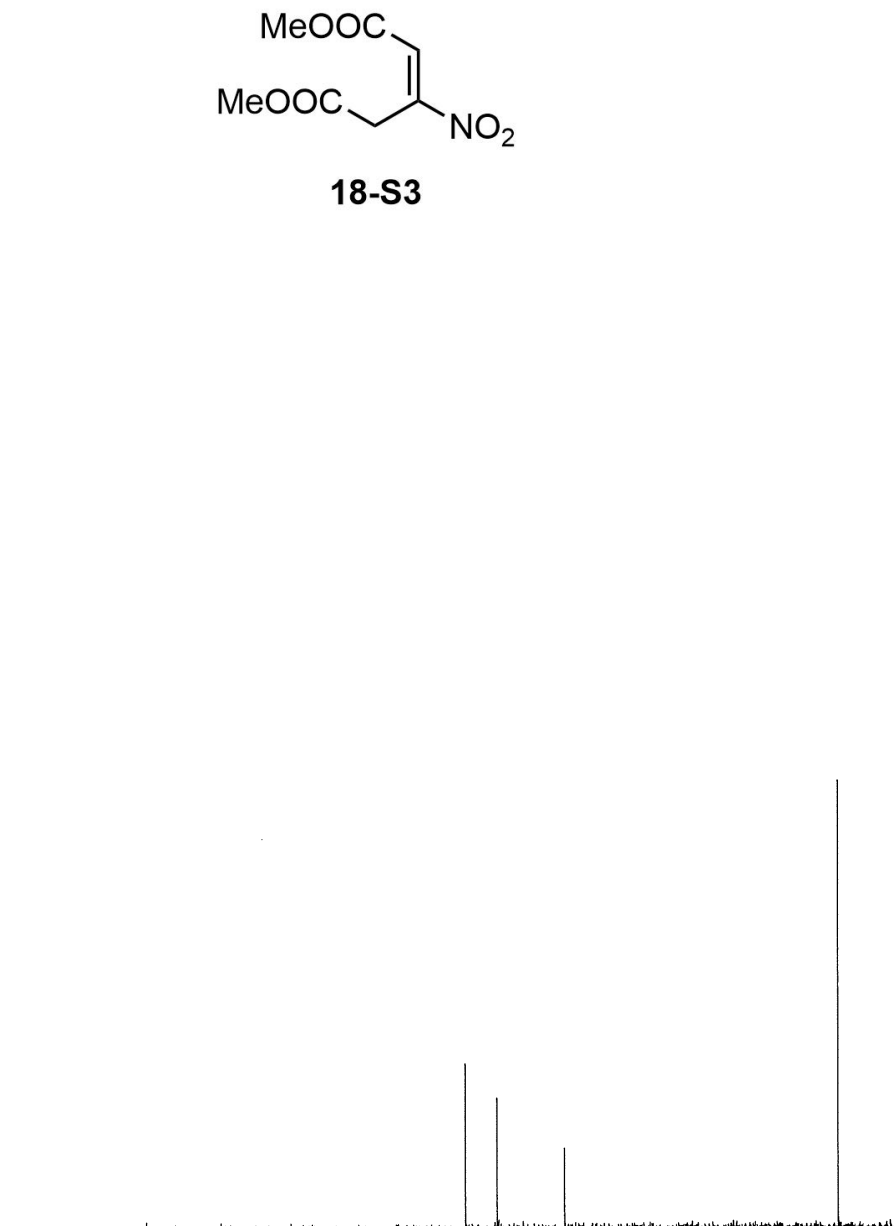

\section{STran}

Current Data Parameters Current Data Parameters NAME EXPNO

F2 - Acquisition Parameter Date_ 20200302 Time INSTRUM PROBHD PULPROG 10.40 spect TD 65536

SOLVENT CDC13

NS

DS

FIDRES

$\mathrm{AQ}$

$\mathrm{RG}$
$\mathrm{DW}$
$\mathrm{DE}$

TE

D 1

D 11

$\mathrm{SFO1}$

$\mathrm{SFO1}$

(⿻一𠃋十

$\mathrm{P} 0$

P 1
P LW1

$\mathrm{SFO} 2$

$\mathrm{NUC} 2$

CPDPRG [ 2

PCPD 2

PLW2

PLW1 2

PLW13 45

$42613.637 \mathrm{~Hz}$

$1.300465 \mathrm{~Hz}$ $0.7689557 \mathrm{sec}$ 203

11.733 usec 18.00 usec $298.2 \mathrm{~K}$

$2.00000000 \mathrm{sec}$ $0.03000000 \mathrm{sec}$

$176.0362620 \mathrm{MHz}$

$13 \mathrm{C}$
3.75 usec 11.25 usec 29.0000 $700.0098000 \mathrm{MHz}$ $1 \mathrm{H}$
waltz 16 65.00 use $13.00000000 \mathrm{~W}$ $0.27768999 \mathrm{~W}$ $0.13982999 \mathrm{~W}$

F2 - Processing parameter SI 131072

SF $\quad 176.0169002 \mathrm{MHz}$ EM

SSB $\quad 0$

$L B$

GB

$1.00 \mathrm{~Hz}$

1.40

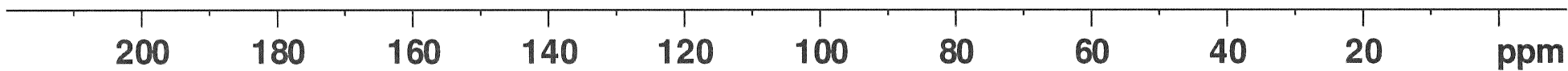


न $\infty$ मी $m$ न $m m N N N H A$

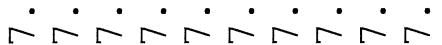
isis $\rightarrow$<smiles>O=C1Cc2ccccc2O1</smiles>

24

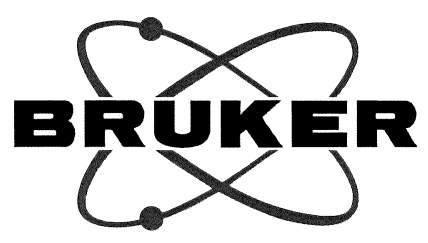

Current Data Parameters NAME

TXPNO

PROCNO

$\mathrm{XZ}-4-15$

F2 - Acquisition Parameter

Date_ 20200120

Time

INSTRUM

PROBHD

PULPROG

$14.16 \mathrm{~h}$

TD

SOLVENT

NS

D $S$

FIDRES

$A Q$

RG

DW

DE

D 1

TD 0

NUC1

P0

PO

P LW1

z151340 spect

-0001

65536

$\mathrm{CDCl} 3$ 16

$10504.202 \mathrm{~Hz}$ $0.320563 \mathrm{~Hz}$ $3.1195135 \mathrm{sec}$ 3.1195135

47.600 usec 20.00 usec $303.2 \mathrm{~K}$

$1.00000000 \mathrm{sec}$ $700.0115500 \mathrm{MHz}$

$1 \mathrm{H}$
3.17 usec 9.50 usec $13.00000000 \mathrm{~W}$

F2 - Processing parameters SI 131072

SF $\quad 700.0070158 \mathrm{MHz}$

WDW

SSB

$L B$

GB

0

$\mathrm{O} \mathrm{Hz}$

PC

1.00

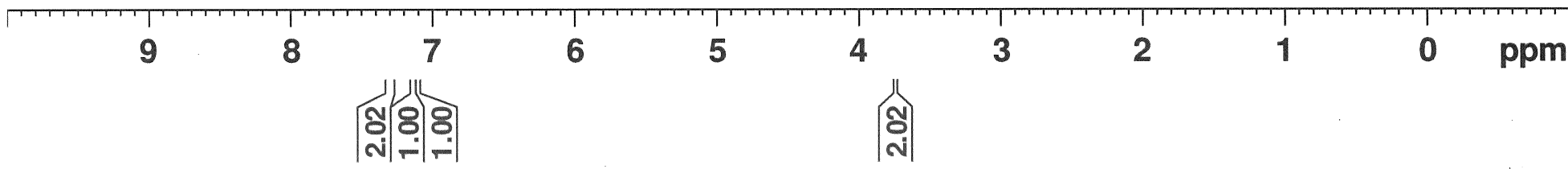




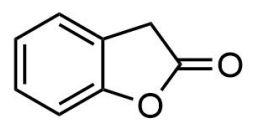

24

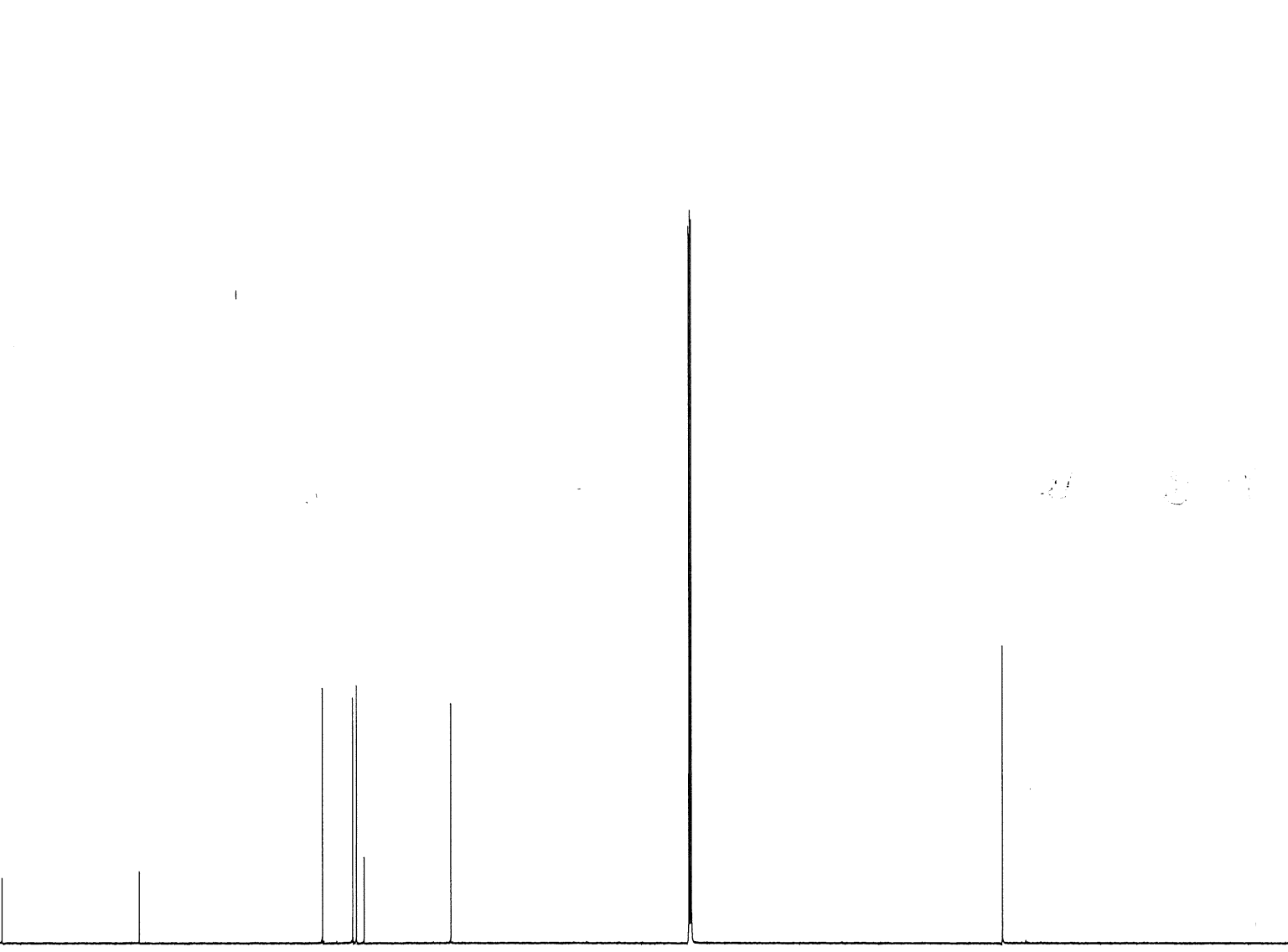

\section{DPR}

Current Data Parameters Current Data Parameters
NAME

NAME

EXPNO

$\mathrm{XZ}-4-157$

F2 - Acquisition Parameter

Date_ 20200120

Time $14.22 \mathrm{~h}$

INSTRUM spect

PROBHD Z151340_0001

PULPROG zgpg30

TD 65536

SOLVENT CDCl3

NS

$S W H$

FIDRES

$\mathrm{AQ}$
$\mathrm{RG}$

DW

$D E$

D 1

D 11

IDO

$\mathrm{SFO1}$

P 0

P LW1

$\mathrm{SFO} 2$

NUC2

CPDPRG [ 2

PCPD 2

PLW2

P LW1 2

59

$$
\begin{array}{r}
59 \\
4
\end{array}
$$

$42613.637 \mathrm{~Hz}$

$1.300465 \mathrm{~Hz}$ $0.7689557 \mathrm{sec}$ 203

11.733 usec 18.00 usec $303.2 \mathrm{~K}$

$2.00000000 \mathrm{sec}$ $0.03000000 \mathrm{sec}$ $176.0362620 \mathrm{MH}$ $176.0362620 \mathrm{MHz}$

3.75 usec 11.25 usec $29.00000000 \mathrm{~W}$ $700.0098000 \mathrm{MHz}$

$$
\begin{gathered}
1 \mathrm{H} \\
\text { waltz } 16
\end{gathered}
$$

65.00 use

$13.00000000 \mathrm{~W}$

$0.27768999 \mathrm{~W}$

$0.13982999 \mathrm{~W}$

2 - Processing parameters $\begin{array}{lc}\text { SI } & 131072 \\ \text { SF } & 176.0169002 \mathrm{MHz}\end{array}$ WDW EM

SSB

LB

$\mathrm{PC}$

0

$1.00 \mathrm{~Hz}$

1.40

\begin{tabular}{ll|l|l|l|l|l|l|l|l|l}
\hline 200 & 180 & 160 & 140 & 120 & 100 & 80 & 60 & 40 & 20 & $p p m$
\end{tabular}




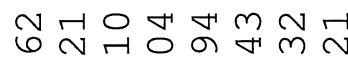

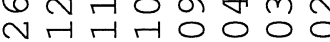

iriririrá

$\longrightarrow$<smiles>Cc1cccc2c1OC(=O)C2</smiles>

25

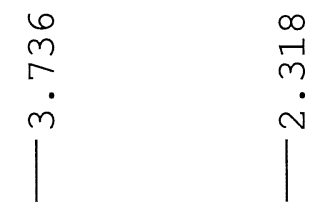

$\infty$
$m$
$m$

N

BriKer

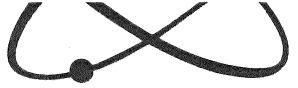

Current Data Parameters NAME

TXP

PROCNO

ROCNO

F2 - Acquisition Parameter

Date_ 20200124

Time-

$17.31 \mathrm{~h}$

INSTRUM

PROBHD

PULPROG

Z151340_0001

TD

SOLVENT

NS

DS

FIDRES

$\mathrm{AQ}$

RG

DW

TE

D 1

TD 0

$\mathrm{SFO1}$

NUC

$\mathrm{P} 0$

P LW1

zg30

$\mathrm{CDCl} 3$

16

$10504.202 \mathrm{~Hz}$

$0.320563 \mathrm{~Hz}$

$3.1195135 \mathrm{sec}$ 195135
25.4

47.600 usec 20.00 usec $303.2 \mathrm{~K}$

$1.00000000 \mathrm{sec}$ $700.0115500 \mathrm{MHz}$

3.17 usec

9.50 usec

$13.00000000 \mathrm{~W}$

F2 - Processing parameters SI 131072

SF $\quad 700.0070149 \mathrm{MHz}$

WDW

$S S B$

$\mathrm{LB}$
$\mathrm{GB}$

PC

$0.30 \mathrm{~Hz}$

1.00

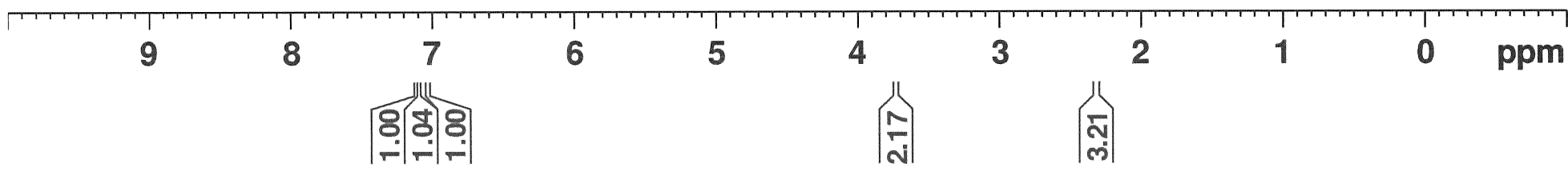


<smiles>O=C1Cc2cccc([N+](=O)[O-])c2O1</smiles>

25
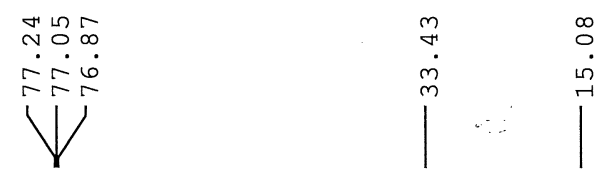

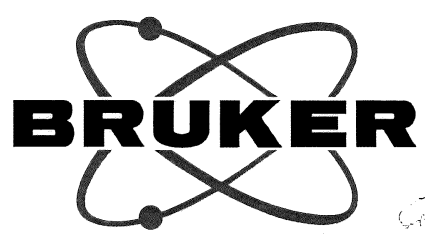

Current Data Parameters

NAME

EXPNO

PROCNO

$\mathrm{XZ}-4-164$

F2 - Acquisition Parameter

Date_ 20200124

Time

INSTRUM

PROBHD

PULPROG

TD

SOLVENT

NS

DS

FIDRES

F ID R

AQ
RG

RG

DW

TE

D 1
D 11

TD 0

$\mathrm{SFO1}$

NUC1

$\mathrm{PO}$

$\mathrm{P} 0$
$\mathrm{P} 1$

P LW1

$\mathrm{SFO} 2$

NUC2

CPDPRG [ 2

PCPD 2

PLW2

P LW1 2 z151340 spect

z $9 p 930$

65536

$\mathrm{CDCl} 3$ 27

$42613.637 \mathrm{~Hz}$ $1.300465 \mathrm{~Hz}$ $0.7689557 \mathrm{sec}$ 203

11.733 usec 18.00 usec $303.2 \mathrm{~K}$

$2.00000000 \mathrm{sec}$ $0.03000000 \mathrm{sec}$

176.0362620 $13 \mathrm{C}$

3.75 usec 11.25 usec $29.00000000 \mathrm{~W}$ $700.0098000 \mathrm{MHz}$$$
\begin{gathered}
1 \mathrm{H} \\
\text { waltz } 16
\end{gathered}
$$

65.00 user $13.00000000 \mathrm{~W}$ $0.27768999 \mathrm{~W}$ $0.13982999 \mathrm{~W}$

F2 - Processing parameters F2 - Processing parameters SF $\quad 176.0169002 \mathrm{MHz}$ WDW $\quad 176.0169002$ SSB LB GB

\begin{tabular}{rrrrrrrrrrr}
\hline & & & & & & & & & & \\
200 & 180 & 160 & 140 & 120 & 100 & 80 & 60 & 40 & 20 & ppm
\end{tabular}




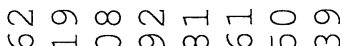

$\mathrm{NH}, \mathrm{H} 0000$

-4
4.45
4.45

$\longrightarrow$

26

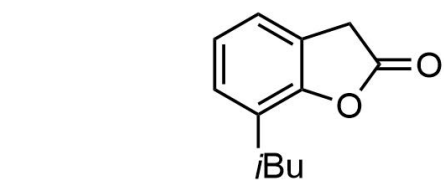

$m \quad m m \omega r-\infty \infty \infty \sigma m \sim \forall m$ ঋ m NNHHHHHH0000 $>$
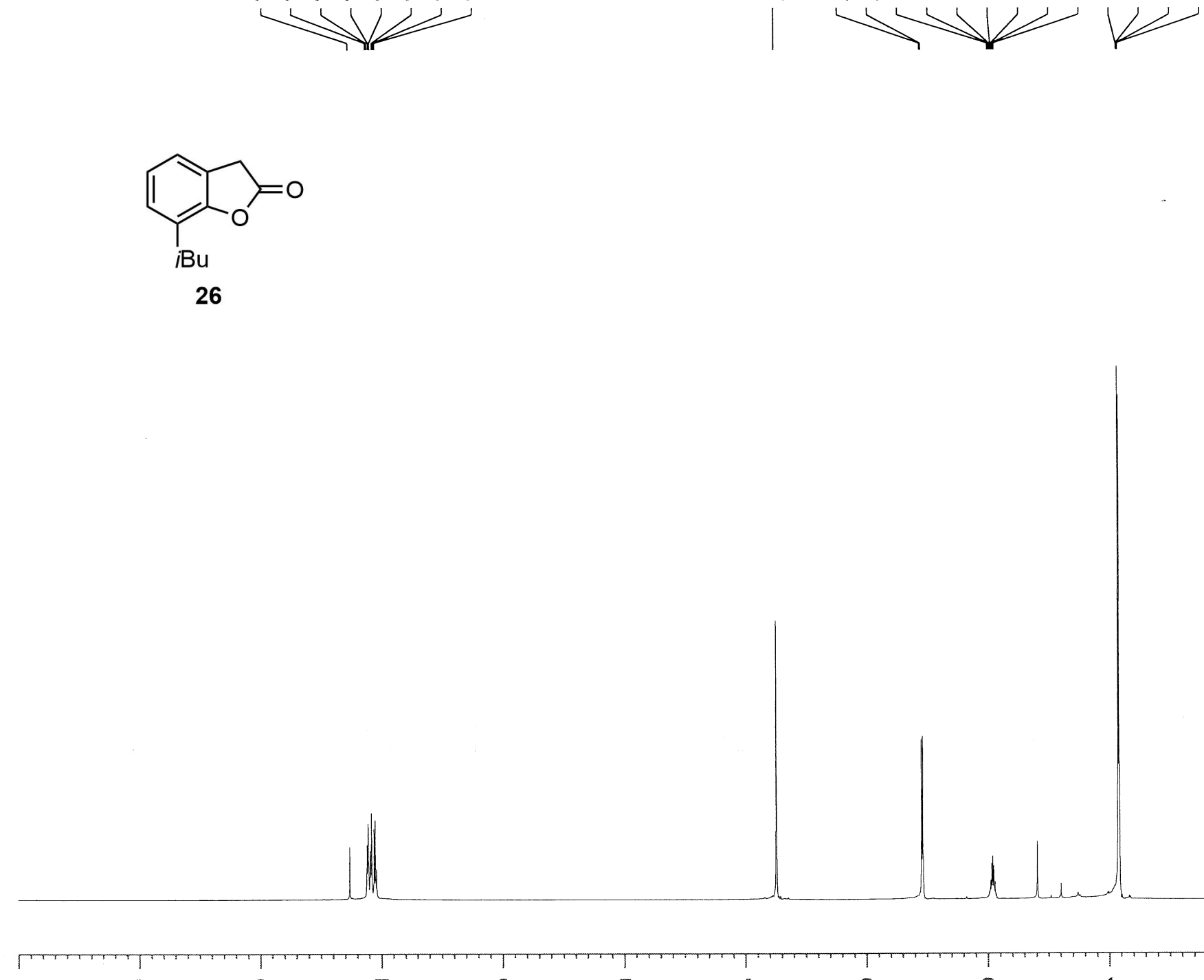

9

8

(1)

cons

65

5

5

\section{BRUKER

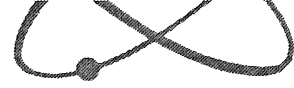 \\ Current Data Parameters \\ NAME \\ EXPNO \\ PROCNO \\ $\mathrm{XZ}-4-256$}

F2 - Acgu

- Acquisition Parameter

20200702

Time $13.48 \mathrm{~h}$

INSTRUM

PROBHD

PULPROG

TD

SOLVENT

NS

DS

SWH

FIDRES

AQ

RG

DW

$\mathrm{DE}$

D 1

TDO

NEO1

PO

$\mathrm{P} 1$

PLW1

2151340_0001

zg30

65536

1

0
$10504.202 \mathrm{~Hz}$

$0.320563 \mathrm{~Hz}$

$3.1195135 \mathrm{sec}$ 25.4

47.600 usec

20.00 usec

$303.2 \mathrm{~K}$

$1.00000000 \mathrm{sec}$

$700.0115500^{1} \mathrm{MHz}$

$1 \mathrm{H}$
3.17 usec

9.50 usec

F2 - Processing parameters

SI 131072

SF $\quad 700.0070170 \mathrm{MHz}$

WDW

SSB

$L B$

PB

EM
0
$0.30 \mathrm{~Hz}$
0
1.00 


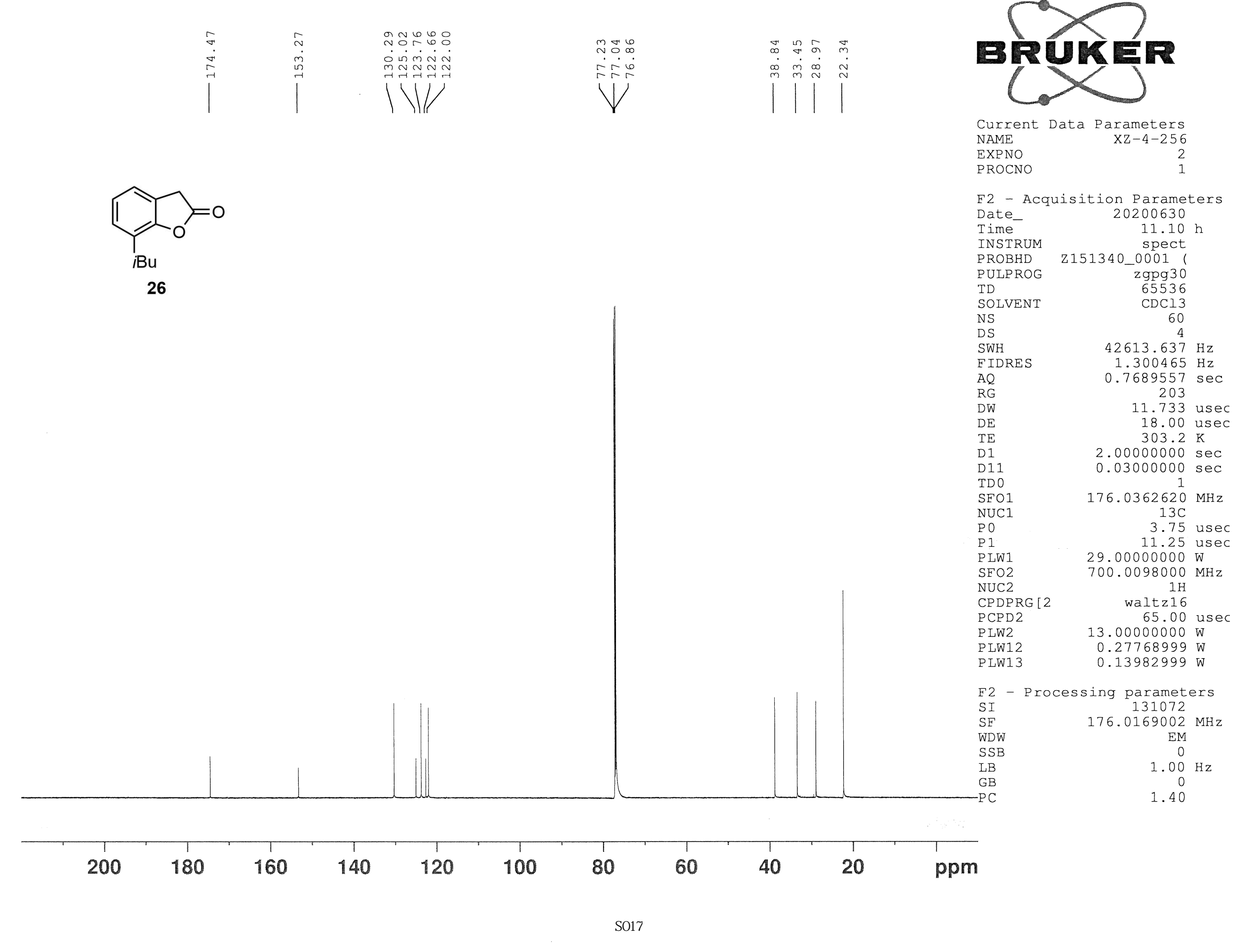


स क

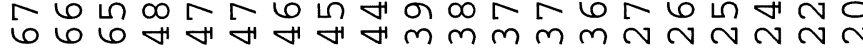

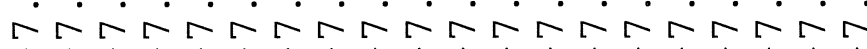

$+2$<smiles>O=C1Cc2cccc(-c3ccccc3)c2O1</smiles>

27

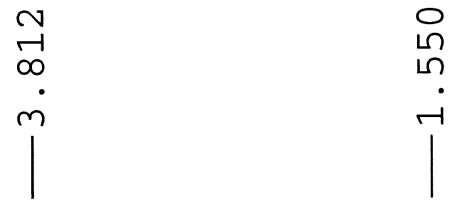

술

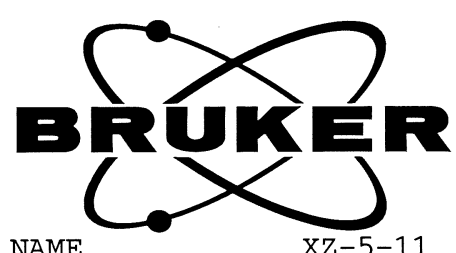

NAME

EXPNO

PROCNO

Date_

Time

INSTRUM

PROBHD

PULPROG

20201109

14.10

TD

SOLVENT

NS

DS

FIDH

$\mathrm{AQ}$

RG

DW

DE

TE

TD0

$5 \mathrm{~mm}$ PABBO BB-

Zg30

CDC13

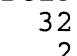

$8278.146 \mathrm{~Hz}$

$0.126314 \mathrm{~Hz}$

$3.9584243 \mathrm{sec}$ 32

60.400 usec 6.50 usec $298.5 \mathrm{~K}$

$1.00000000 \mathrm{sec}$ NUC1

PL1

PL1W

SFO1

$1 \mathrm{H}$
11.20 usec
$0.00 \mathrm{~dB}$
$10.27361584 \mathrm{~W}$
$400.1424710 \mathrm{MHz}$
$131072 \mathrm{MHz}$
$400.1400083 \mathrm{MHz}$
$\mathrm{EM}$
0
$0.30 \mathrm{~Hz}$
0
1.00

$\begin{array}{ll}9 & 8\end{array}$

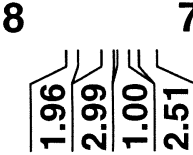

6

5

4
กิ
N̦ 


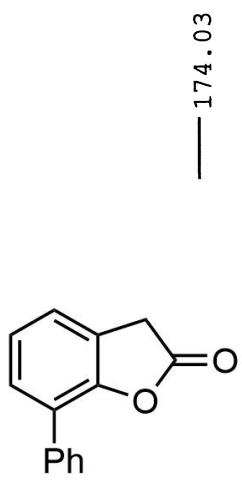

27

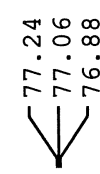

i.

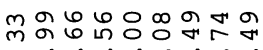

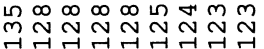

WW
3.

Current Data Parameters

NAME

EXPNO

PROCNO

F2 - Acquisition Parameters

Date 20201109

Time $\quad 13.47$

INMT

TNSTRUM

PROBHD

TD

TD

NS

DS

SWH

FIDRES

AQ

DW
DE
TE

TE

D1
D11
TD0

SFO1

NUC1

PO

P1

PLW1

$\mathrm{SFO} 2$

CPDPRG [2

PCPD2

PCPD2

PLW12

PLW13

2151340_0001

zgpg 30
65536

$\mathrm{CDCl} 3$

5
4

$42613.637 \mathrm{~Hz}$

$1.300465 \mathrm{~Hz}$

$0.7689557 \mathrm{sec}$ 203

11.733 usec

18.00 usec

$298.2 \mathrm{~K}$

$2.00000000 \mathrm{sec}$

$0.03000000 \mathrm{sec}$

$176.0362620 \mathrm{MHz}$ $13 \mathrm{C}$

3.75 usec

$29.00000000 \mathrm{~W}$

$700.0098000 \mathrm{MHz}$

$$
\text { waltz16 }
$$

65.00 usec

$13.00000000 \mathrm{~W}$

$13.00000000 \mathrm{~W}$
$0.27768999 \mathrm{~W}$

$0.13982999 W$

F2 - Processing parameters

SI

SF $\quad 176.0169002 \mathrm{MHz}$

WDW

SSB

LB

GB

EM

EM
0
$1.00 \mathrm{~Hz}$
0
1.40


on $\infty \pi r r \sim m m$

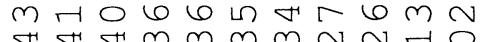
$\pi$ m brararararar $\longrightarrow$<smiles>Cc1cc2c(cc1-c1ccccc1)CC(=O)O2</smiles>

28

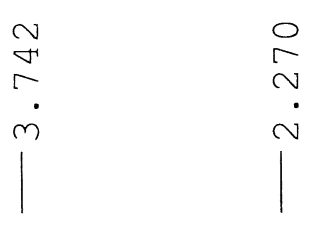

BR UKER

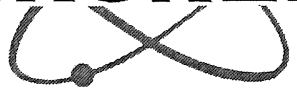

Current Data Parameters NAME

EXPNO

PROCNO

F2 - Acquisition Parameters Date__ 20200316

Time $\quad 16.58 \mathrm{~h}$

INSTRUM

PROBHD

PULPROG

Z151340 spect

TD

SOLVENT

NS

DS

FIDRES

AQ

DW

$\mathrm{DE}$

D 1

TD 0

$\mathrm{SFO} 1$

NUC1

$\mathrm{P} 1$
$\mathrm{PLW} 1$

$\mathrm{zg} 30$
65536

2
$10504.202 \mathrm{~Hz}$

$0.320563 \mathrm{~Hz}$

$3.1195135 \mathrm{sec}$ 28.5

47.600 usec

20.00 usec

$298.2 \mathrm{~K}$

$1.00000000 \mathrm{sec}$

$700.0115500 \mathrm{MHz}$

$1 \mathrm{H}$

3.17 usec

.50 usec

F2 - Processing parameters

SI 131072

SF $\quad 700.0070174 \mathrm{MHz}$

WDW

SSB

LB

GB
EM
0
$0.30 \mathrm{~Hz}$
0
1.00 


\section{SRUKER}

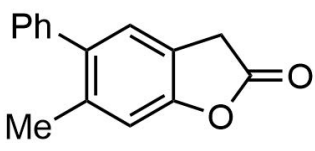

28

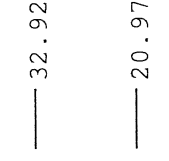

$(>-1)$

Current Data Parameters

NAME

EXPNO

PROCNO

F2 - Acquisition Parameters

Date_ 20200316

Time 17.05

INSTRUM spect

PROBHD Z151340_0001

PULPROG zgpg30

TD

SOLVENT

NS

$\mathrm{CDCl} 3$

DS

SWH $\quad 42613.637 \mathrm{~Hz}$

FIDRES $\quad 1.300465 \mathrm{~Hz}$

$\mathrm{AQ} \quad 0.7689557 \mathrm{sec}$

AQ

$D W$

DW

$\mathrm{DE}$

D1

D11

TD 0
SFO1

$\mathrm{SFO} 1$
$\mathrm{NUC1}$

NeI

$\mathrm{P} 1$

PLW1

$\mathrm{SFO} 2$

$\mathrm{SFO} 2$

CPDPRG

CPDPR

PCPD2

PLW12

PLW13

1.733 usec

18.00 usec

$298.2 \mathrm{~K}$

$2.00000000 \mathrm{sec}$

$0.03000000 \mathrm{sec}$

$176.0362620 \mathrm{MHz}$ $13 \mathrm{C}$

3.75 usec

11.25 usec

$29.00000000 \mathrm{~W}$

$700.0098000 \mathrm{MHz}$

$$
\begin{array}{r}
1 \mathrm{H} \\
\text { waltz16 }
\end{array}
$$

65.00 usec

$13.00000000 \mathrm{~W}$

$0.27768999 \mathrm{~W}$

$0.13982999 \mathrm{~W}$

F2 - Processing parameters

SI 131072

SF $\quad 176.0169002 \mathrm{MHz}$

WDW EM

$\begin{array}{ll}\mathrm{SSB} & 1.00 \mathrm{~Hz}\end{array}$

GB

0
1.40 


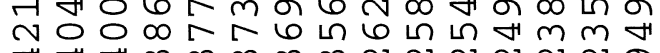
$\forall \forall \forall m m m m \sim N \sim \sim N$

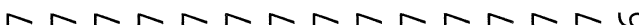
$\ldots$<smiles>CCc1c(-c2ccccc2)cc2c(c1C)OC(=O)C2</smiles>

29
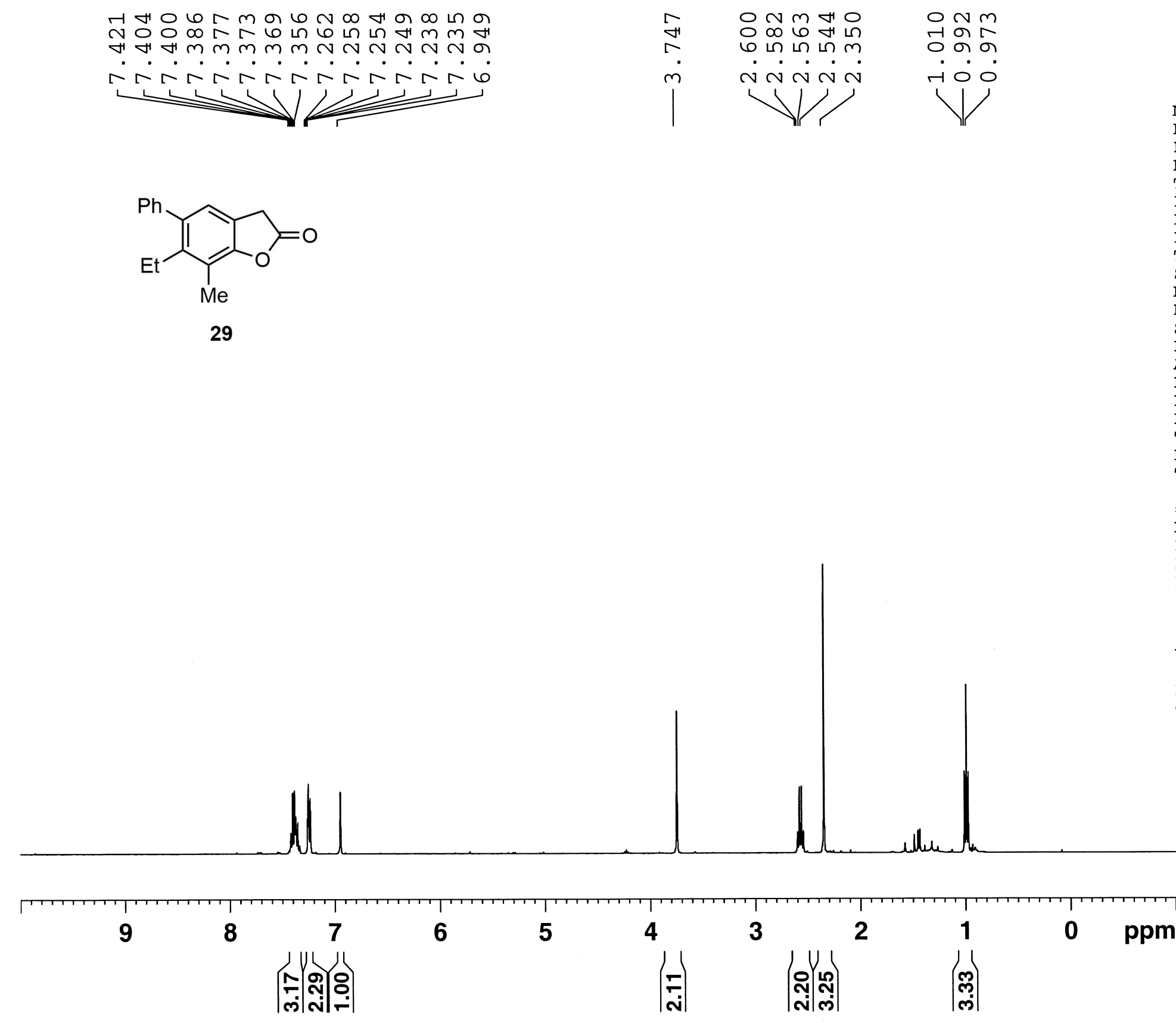

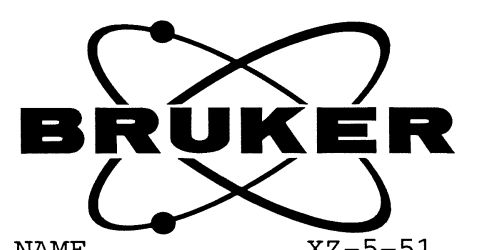

NAM

EXPNO

PROCNO

Date_

Time

INSTRUM

PROBHD

PULPROG

SOLVENT

NS

SWH

FIDRES

AQ

RG

$\mathrm{DW}$

$\mathrm{DE}$

$\mathrm{D} 1$

TDO

20201109

15.08

spect

$5 \mathrm{~mm}$ РABBO BB-

$\mathrm{zg} 30$

6536

$\mathrm{CDCl} 3$

$8278.146 \mathrm{~Hz}$

$0.126314 \mathrm{~Hz}$

$3.9584243 \mathrm{sec}$

90.5

60.400 usec

6.50 us

$1.00000000 \mathrm{sec}$

$========$ CHANNEL $\mathrm{f} 1$ ========= NUC1

P1

PL1

PL1W

SFO1

SF
WDW

SSB

LB

GB

$\mathrm{PC}$

10.75 usec $-4.00 \mathrm{~dB}$ $25.80615616 \mathrm{~W}$ $400.1424710 \mathrm{MHz}$ 131072

$400.1400083 \mathrm{MHz}$

EM

0

$0.30 \mathrm{~Hz}$

1.00 
<smiles>CCc1c(-c2ccccc2)cc2c(c1C)OC(=O)C2</smiles>

29
Current Data Parameters

NAME

EXPNO

PROCNO

F2 - Acquisition Parameters

Date__ 20201109

Time $13.32 \mathrm{~h}$

INSTRUM

PROBHD

PULPROG Z zgpg30

z151340 spect

TD

SOLVENT

NS

DS

SWH

FIDRES

AQ

RG

DW

DE

TE

D1

TD 11

TD 0

SFO1

PO

$\mathrm{P} 1$

PLW1

$\mathrm{SFO} 2$

NUC2

CPDPRG [2

PCPD2

PLW12

PLW13

F2 - Processing parameters

SI 131072

SF $\quad 176.0169002 \mathrm{MHz}$

WDW

SSB

LB

GB 
$\infty \infty \sim m \sim \sim \sim m \sim \infty$ भ - memen rrarrara

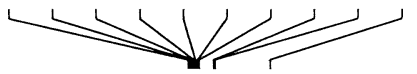

30

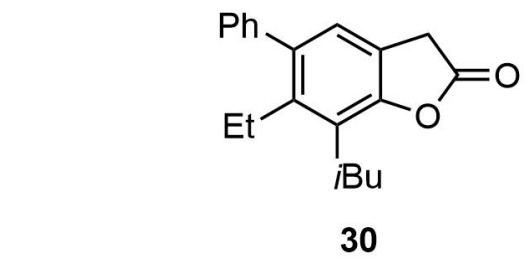

の $\infty$ म

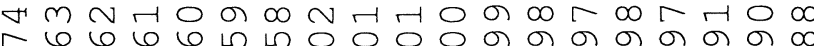
$\dot{m} \dot{\sim} \dot{\sim} \dot{\sim} \dot{\sim} \dot{\sim} \dot{\sim} \dot{\sim} \dot{H} \dot{H} \dot{0} \dot{0} \dot{0} \dot{0}$
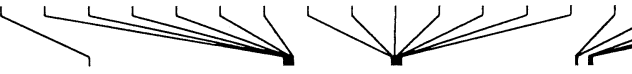

\section{BRUKER \\ (n)}

Current Data Parameters NAME $\quad \mathrm{XZ}-4-257$ EXPNO

PROCNO

F2 - Acquisition Parameters

Date 20200702

Time $14.10 \mathrm{~h}$

INSTRUM spect

PROBHD Z151340_0001

PUIPROG Z151340_0001

$\begin{array}{lr}\text { PULPROG } & \text { Zg30 } \\ \text { TD } & 65536\end{array}$

$\begin{array}{ll}\text { TD } & 65536 \\ \text { SOLVENT } & \text { CDC13 }\end{array}$

$\begin{array}{lr}\text { SOLVENT } & \text { CDCl3 } \\ \text { NS } & 16\end{array}$

DS

SWH

FIDRES

$\mathrm{AQ}$
$\mathrm{RG}$

DW

$\mathrm{DE}$

TE

TD 0

$\mathrm{SFO} 1$

NUC1

PO

P 1

\section{$10504.202 \mathrm{~Hz}$}

$0.320563 \mathrm{~Hz}$

$3.1195135 \mathrm{sec}$

47.600 usec

20.00 usec

$303.2 \mathrm{~K}$

$1.00000000 \mathrm{sec}$

$700.0115500 \mathrm{MHz}$

$1 \mathrm{H}$

3.17 usec

9.50 usec

$13.00000000 \mathrm{~W}$

F2 - Processing parameters SI 131072

SF $\quad 700.0070168 \mathrm{MHz}$

WDW

WDW

SSB

LB

$\mathrm{GB}$
$\mathrm{PC}$

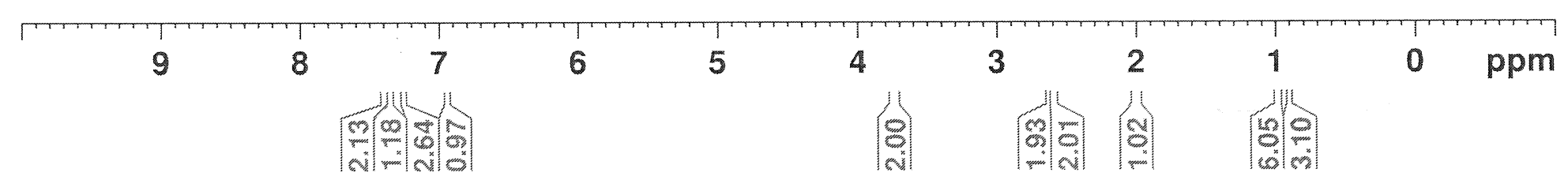




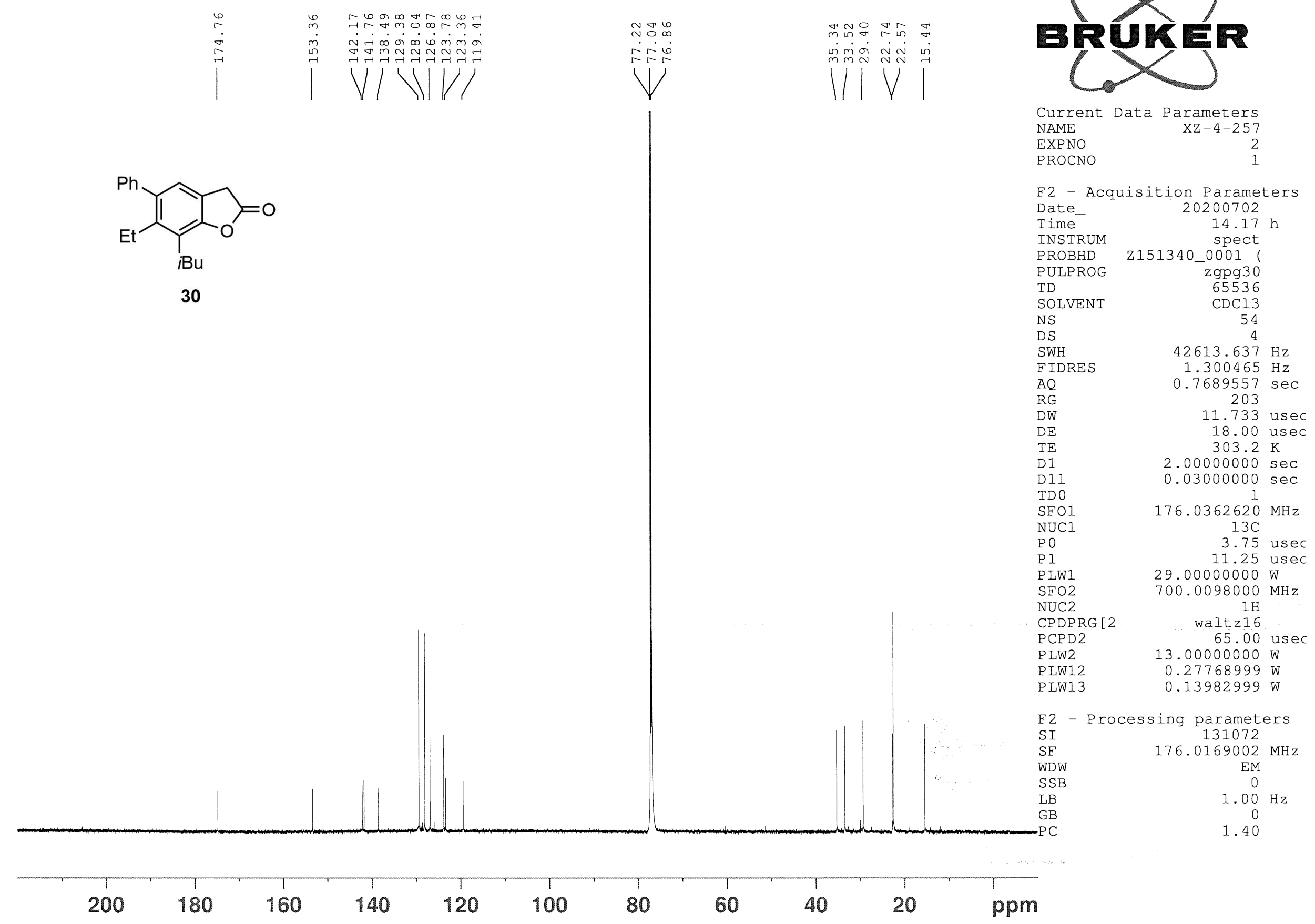




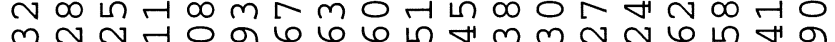

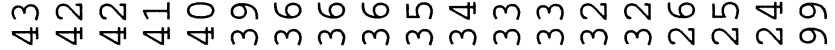

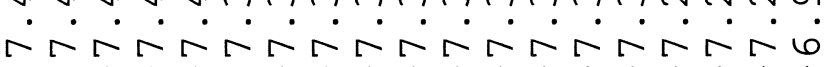

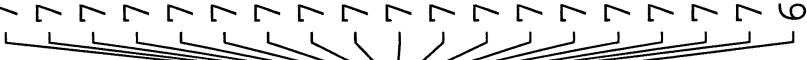
II

31

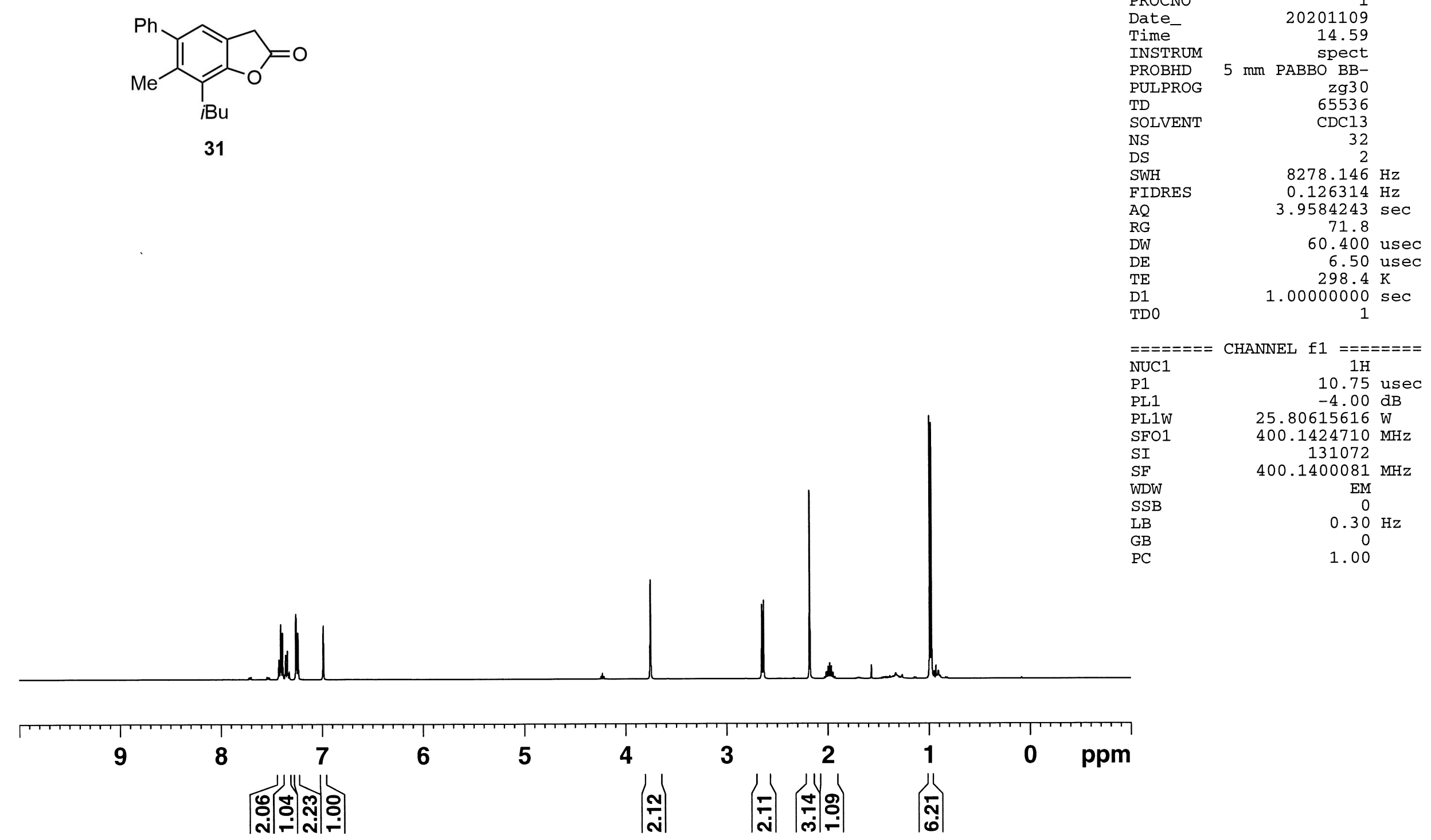

H $\ln \infty N$ N त (

m N N N $N$ N

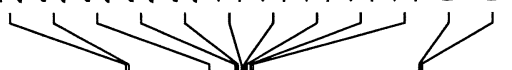




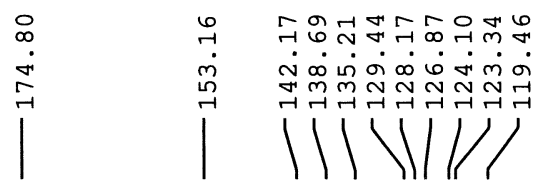<smiles>Cc1c(-c2ccccc2)cc2c(c1CC(C)C)OC(=O)C2</smiles>

31
สํํำ ำ $\stackrel{n}{m} \underset{m}{\infty} \stackrel{\infty}{\sim}$ 1111

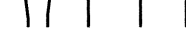

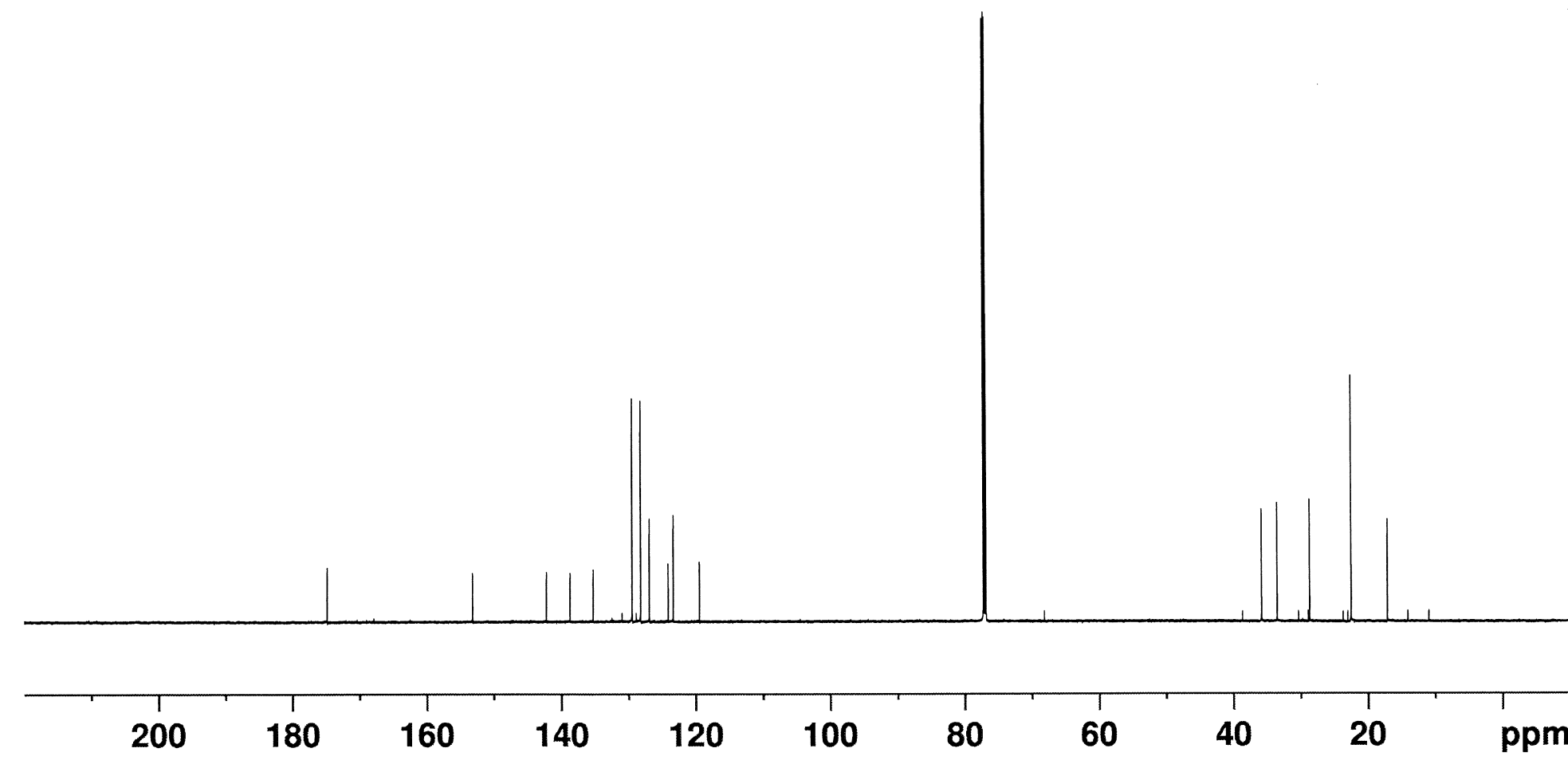

\section{BR}

Current Data Parameter NAME $\mathrm{XZ}-4-170$

EXPNO

PROCNO

F2 - Acquisition Parameters

Date_ 20201109

Time $\quad 13.24 \mathrm{~h}$

INSTRUM spect

PROBHD Z151340 0001

PULPROG ZgPg30

TD

65536

SOLVENT

NS

8

DS

SWH

FIDRES

$\mathrm{AQ}$

RG

DW

TE

D1

D11

TDO 1

NUC1

$\mathrm{P} 1$

PLW1

$\mathrm{SFO} 2$

NUC2

CPDPRG [2

PCPD2

PLW2

PLW12

PLW13

$42613.637 \mathrm{~Hz}$

$1.300465 \mathrm{~Hz}$

$0.7689557 \mathrm{sec}$$$
203
$$

11.733 usec

18.00 usec

$2.00000000 \mathrm{sec}$

$2.00000000 \mathrm{sec}$ $0.03000000 \mathrm{sec}$

$176.0362620 \mathrm{MHz}$ $13 \mathrm{C}$

3.75 usec
11.25 usec

$29.00000000 \mathrm{~W}$

$700.0098000 \mathrm{MHz}$

$$
\begin{array}{r}
1 \mathrm{H} \\
\text { waltz } 16
\end{array}
$$

65.00 usec

$13.00000000 \mathrm{~W}$

$0.27768999 \mathrm{~W}$

$0.13982999 \mathrm{~W}$

F2 - Processing parameters

SI

SF $\quad 176.0169002 \mathrm{MHz}$

WDW

SSB

LB

$\mathrm{GB}$
$-\mathrm{PC}$
EM
0
$1.00 \mathrm{~Hz}$
0
1.40

100

60

.


N

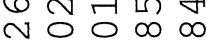

둫

111

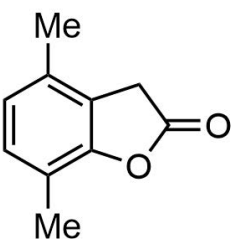

32

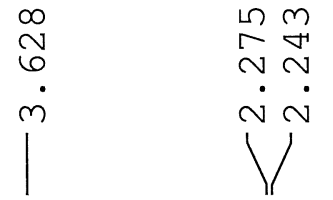

Current Data Parameters

NAME x $-4-216$

$$
\text { EXPNO }
$$

$\mathrm{XZ}-4-21$

PROCNO$$
1
$$

F2 - Acquisition Parameter

Date_ 20200302

Time $17.12 \mathrm{~h}$

INSTRUM

PROBHD

spect

Z151340_0001

TD

SOI

NS

D S

FIDRE

$\mathrm{AQ}$

RG

DW

$\mathrm{DE}$

TE

D1

SFO1

NEO1

$\mathrm{P} 0$

P 1
P L W 1

zg30

16
2

$10504.202 \mathrm{~Hz}$

$0.320563 \mathrm{~Hz}$

$3.1195135 \mathrm{sec}$ 28.5

47.600 usec 20.00 usec $298.2 \mathrm{~K}$

$1.00000000 \mathrm{sec}$

$700.0115500 \mathrm{MHz}$

3.17 usec 9.50 usec

$13.00000000 \mathrm{~W}$

F2 - Processing parameters

SI 131072

SF $\quad 700.0070151 \mathrm{MHz}$

WDW

SSB

EM

$0.30 \mathrm{~Hz}$

GB

$\mathrm{PC}$

1.00

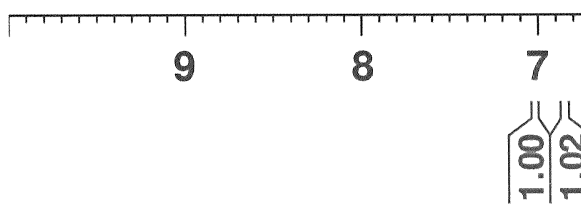

6

5

4

2

ppm

잉요인

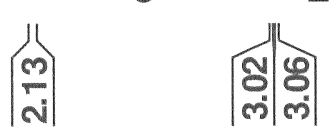




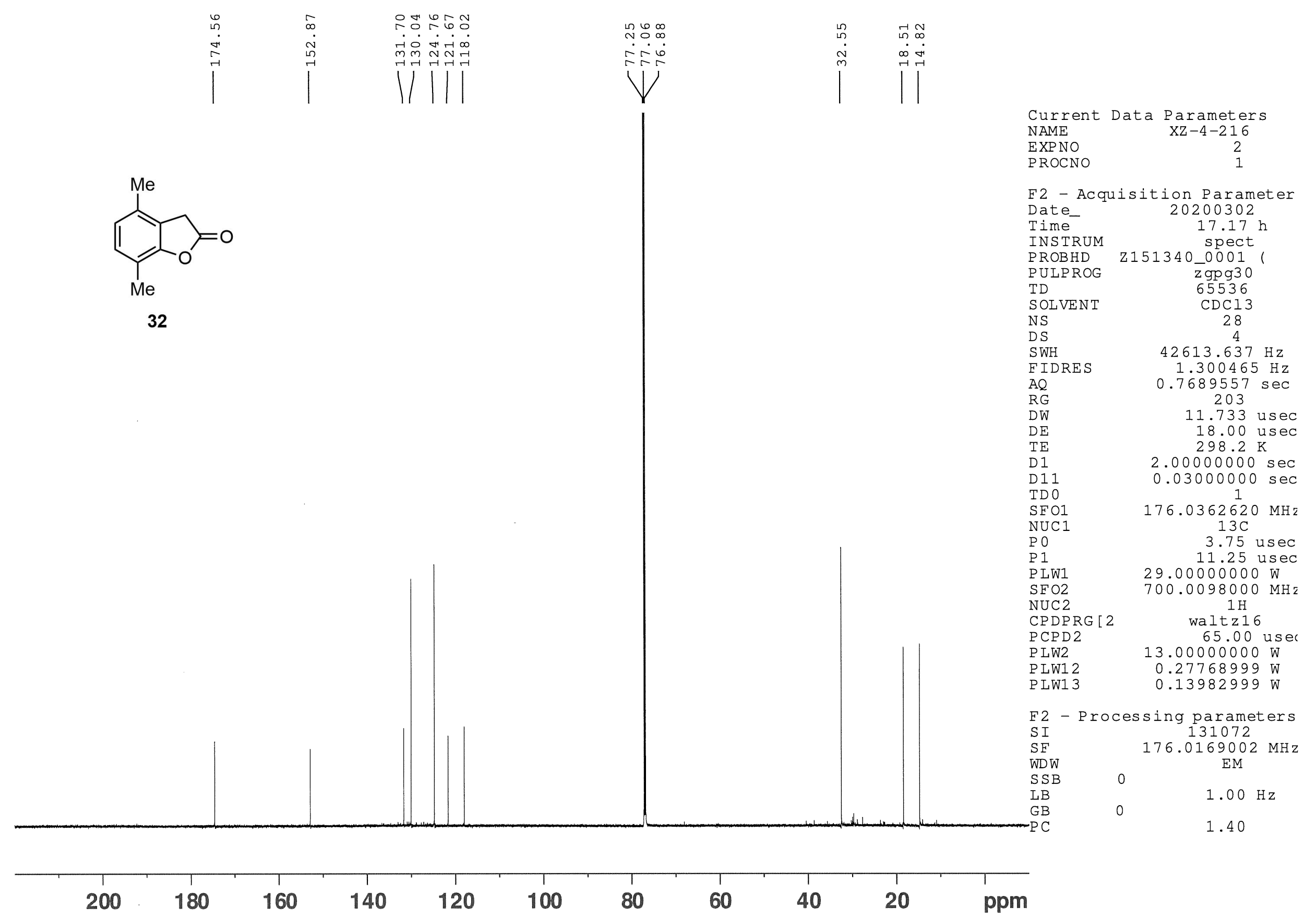


$\begin{array}{lll}1 & 0 & 0\end{array}$

৩のの $\infty$

-

11

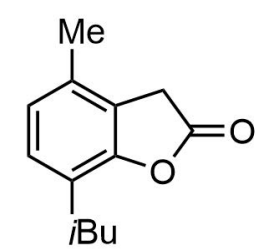

33 सm ○ा आम मत बन बन ब $\infty$ व N $\mathrm{NH}$ मHमHनि

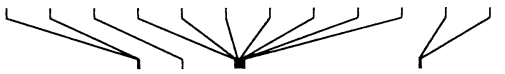

\section{BRUKER

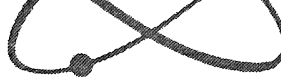

Current Data Parameters NAME

EXPNO

PROCNO

F2 - Acquisition Parameters Date_ 20200702

Time $\quad 14.29 \mathrm{~h}$

INSTRUM spect

PROBHD Z151340_0001

PULPROG Zg30

$\begin{array}{lr}\text { PULPROG } & \mathrm{zg} 30 \\ \text { TD } & 65536\end{array}$

SOLVENT CDCl3

NS

DS

SWH

FIDRES

AQ

DW

DE

TE

D1

TD 0

SFO1

PO

P 1
PLW1

16

$10504.202 \mathrm{~Hz}$ $0.320563 \mathrm{~Hz}$

$3.1195135 \mathrm{sec}$ 28.5

47.600 usec

20.00 usec

$303.2 \mathrm{~K}$

$1.00000000 \mathrm{sec}$

$700.0115500 \mathrm{MHz}$

$1 \mathrm{H}$

$1 \mathrm{H}$
3.17 usec

9.50 usec

$13.00000000 \mathrm{~W}$

F2 - Processing parameters

SI 131072

SF $\quad 700.0070167 \mathrm{MHz}$

$\begin{array}{cc}\text { WDW } & \text { EM } \\ \text { SSB } & 0\end{array}$

LB $\quad 0.30 \mathrm{~Hz}$

$\begin{array}{lr}\mathrm{GB} & 0 \\ \mathrm{PC} & 1.00\end{array}$

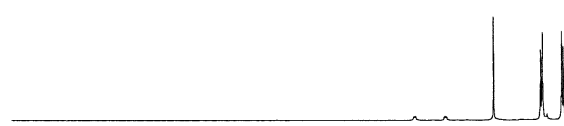

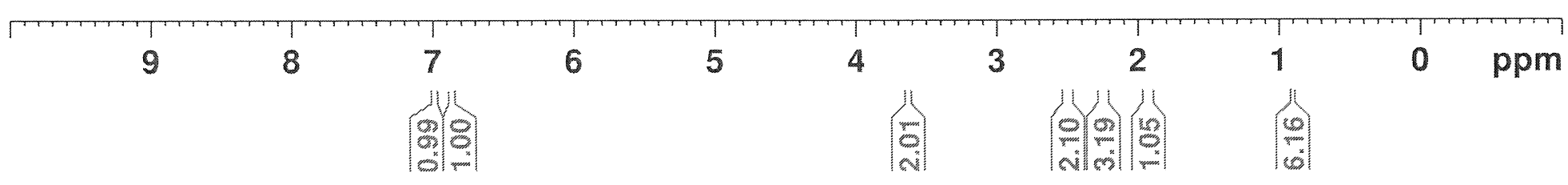




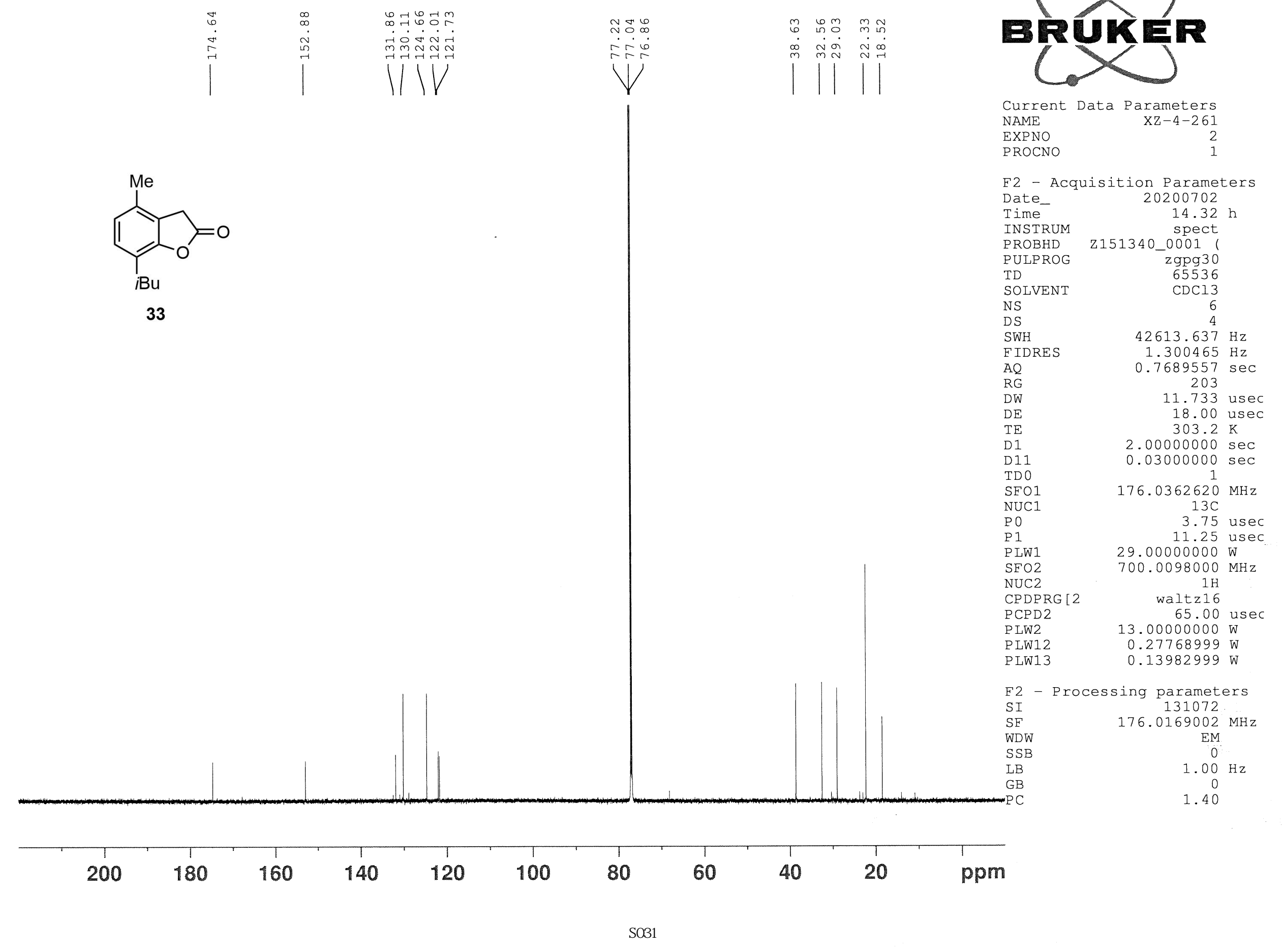



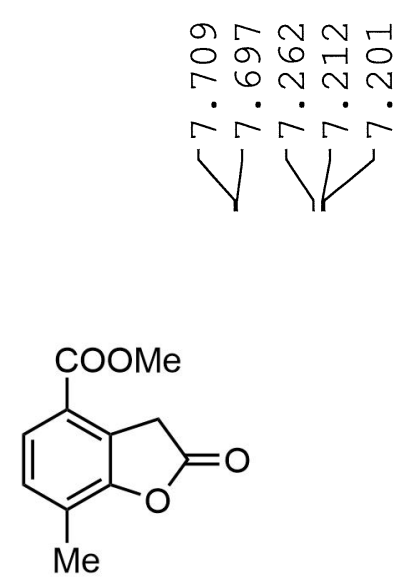

34

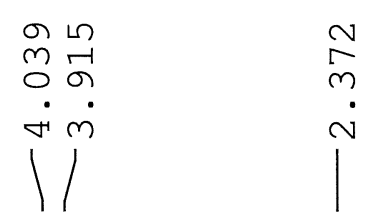

$\stackrel{N}{i}$

Current Data Parameter

NAME $\mathrm{XZ}-4-217$

PROCNO

1

F2 - Acquisition Parameter

Date_ 20200302

Time $\quad 17.02 \mathrm{~h}$

INSTRUM spect

PROBHD Z151340_0001

PULPROG zg30

TD 65536

$\begin{array}{lr}\text { NS } & \text { CDC1 } \\ \text { NS } & 16\end{array}$

D

SWH

FIDRES

$\mathrm{AQ}$

$10504.202 \mathrm{~Hz}$

$0.320563 \mathrm{~Hz}$

$3.1195135 \mathrm{sec}$ 28.5

47.600 usec 20.00 usec $298.2 \mathrm{~K}$

$1.00000000 \mathrm{sec}$

$1.00000000 \mathrm{sec}$

$700.0115500 \mathrm{MHz}$ $1 \mathrm{H}$

3.17 usec

$13.00000000 \mathrm{~W}$

$\begin{array}{ll}\mathrm{P} & 0 \\ \mathrm{P} & 1\end{array}$

PLW1

F2 - Processing parameters
SI
131072

SF $\quad 700.0070151 \mathrm{MHz}$

WDW

S S B

EM

EM

GB

$0.30 \mathrm{~Hz}$

PC

1.00 


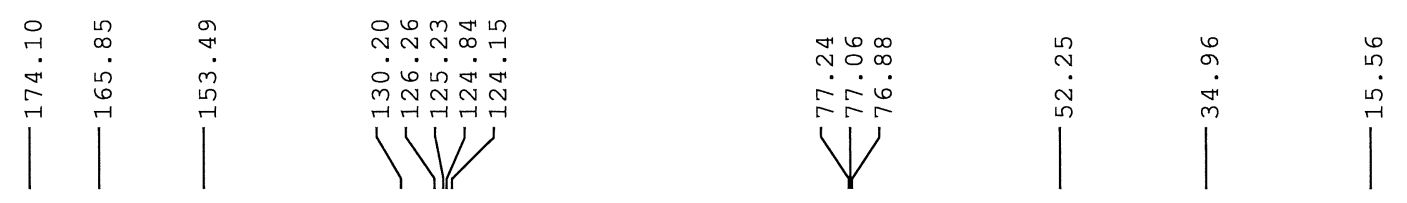
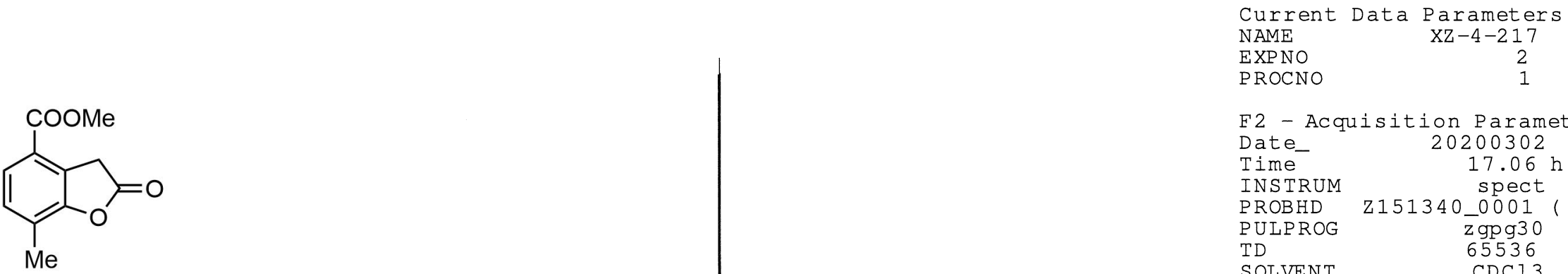

34

151340 pect
F2 - Acquisition Parameter Date_ 20200302

Time

INSTRUM

PROBHD

PULPROG $17.06 \mathrm{~h}$

TD

SOLVENT

SOI
NS
DS

SWH

FIDRES

$\mathrm{AQ}$

$R G$

DW

$\mathrm{DE}$

D 1

D 11

TD 0

SFO1

NUC1

P 0

P 1

P LW1

$\mathrm{SFO} 2$

$\mathrm{NUC} 2$

CPDPRG [ 2

PCPD 2

P IT2

P LW1 2

PLW1 3

00011

z gpg30
65536

31

$42613.637 \mathrm{~Hz}$

$1.300465 \mathrm{~Hz}$ $0.7689557 \mathrm{sec}$ 203

11.733 usec 18.00 usec $298.2 \mathrm{~K}$

$2.00000000 \mathrm{sec}$ $0.03000000 \mathrm{sec}$

$176.0362620 \mathrm{MHz}$ $13 \mathrm{C}$

3.75 usec 11.25 usec $29.00000000 \mathrm{~W}$ $700.0098000 \mathrm{MHz}$

$$
\begin{gathered}
1 \mathrm{H} \\
\text { waltzi } 16
\end{gathered}
$$

65.00 use

$13.00000000 \mathrm{~W}$

$0.27768999 \mathrm{~W}$

$0.13982999 \mathrm{~W}$

F2 - Processing parameters SI 131072 SF $\quad 176.0169002 \mathrm{MHz}$ WDW EM

SSB

GB
0

0
$1.00 \mathrm{~Hz}$
1.40

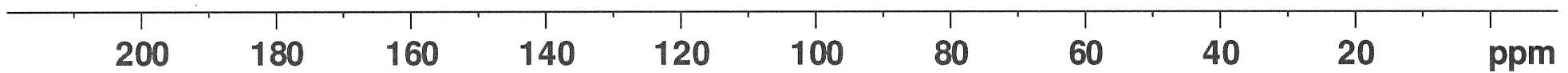



$m$
0
$\infty \infty \sim \sim-$
$\therefore r \dot{r} \cdot \dot{0}$
$1>11$

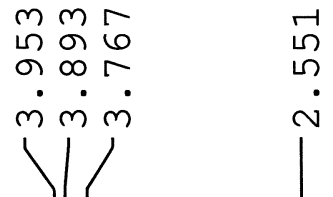
11

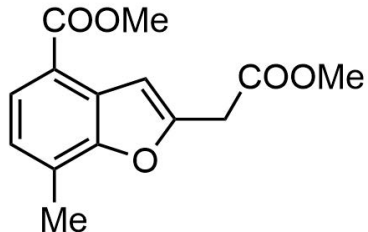

35

i

\section{BR $(><)$}

Current Data Parameters NAME

EXPNO

PROCNO

F2 - Acquisition Parameters

Date 20201201

$\begin{array}{lr}\text { Time } & 16.10 \mathrm{~h}\end{array}$

PROBHD Z151340_0001

PROBHD Z151340_0001

$\mathrm{zg} 30$
TD

$\begin{array}{ll}\text { TD } & 65536 \\ \text { SOLVENT } & \text { CDC13 }\end{array}$

DS

SWH

FIDRES

AQ

RG

DW

DE

TE

TD 1

TD0

NEO1

PO

P1

PLW1

16

$10504.202 \mathrm{~Hz}$

$0.320563 \mathrm{~Hz}$

$3.1195135 \mathrm{sec}$ 40.3

47.600 usec

20.00 usec

$298.2 \mathrm{~K}$

$1.00000000 \mathrm{sec}$

$700.0115500 \mathrm{MHz}$

$1 \mathrm{H}$

3.17 usec

13.00000000 usec

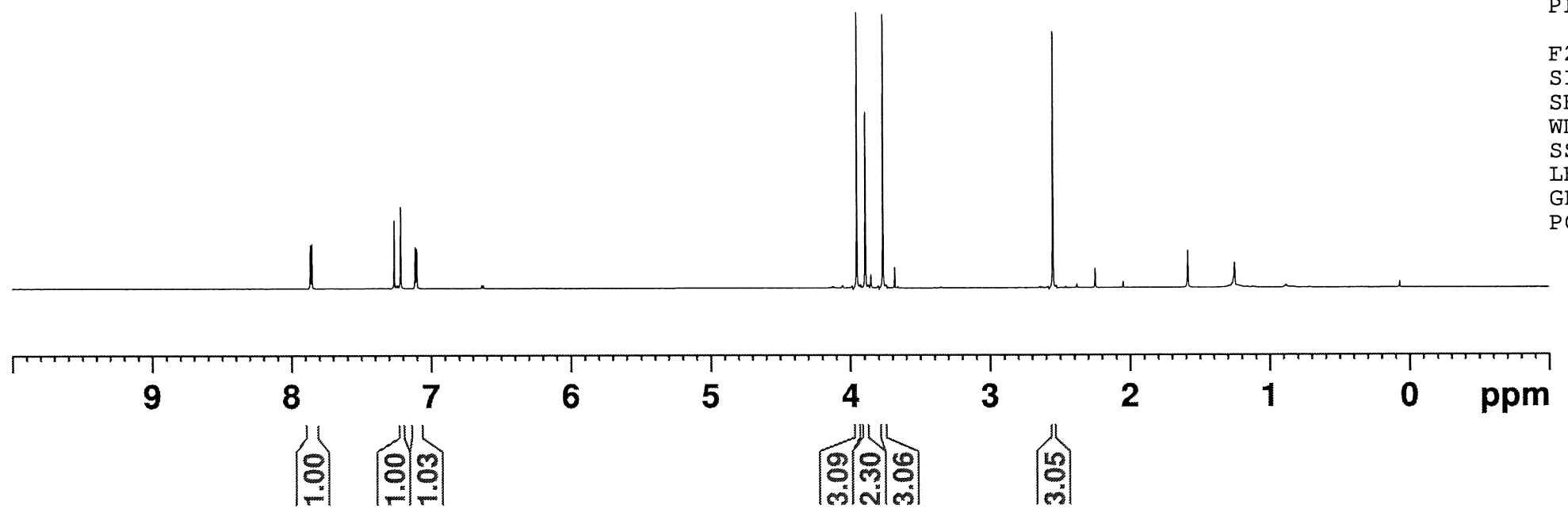

2 - Processing parameters

SI

$700.0070154 \mathrm{MH}$

SFW

EM

$\begin{array}{ll}\mathrm{LB} & 0.30 \mathrm{~Hz}\end{array}$

GB

$0.30 \mathrm{~Hz}$

1.00 

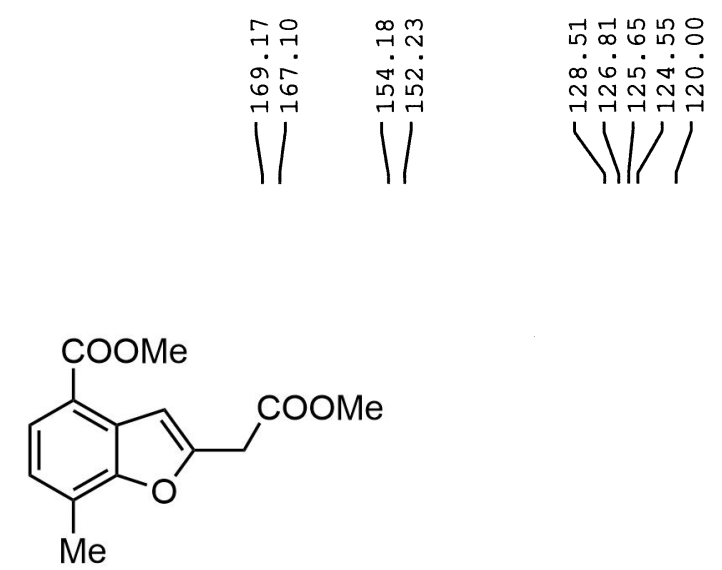

35

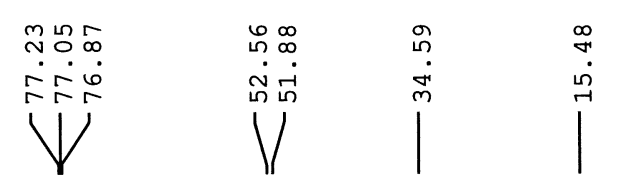

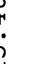

$$
\text { i }
$$

BRUE

Current Data Parameters NAME XZ-5-146 EXPNO PROCNO

F2 - Acquisition Parameters Date__ 20201201 Time $\quad 16.14 \mathrm{~h}$

INSTRUM spect

INSTRUM Z151340_0001

PULPROG Z151340_001

$\begin{array}{lr}\text { PULPROG } & \text { zgpg30 } \\ \text { TD } & 65536\end{array}$

SOLVENT

NS

DS

SWH

FIDRES

AQ

RG

RG
DW
DE
$T E$

$\mathrm{TE}$

D11

TD 0

NFO1

$\mathrm{NO}$

P1

PLW1

$\mathrm{SFO} 2$

NUC2

CPDPRG [ 2

CPDPRG

PCPD2

PLW12
PLW13

F2 - Processing parameters

SI 131072

SF $\quad 176.0169002 \mathrm{MHz}$

WDW

SSB

LB

GB

EM
0
$1.00 \mathrm{~Hz}$
0
1.40




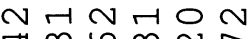

ब

riririr

111

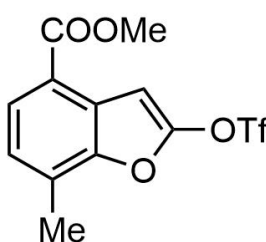

36

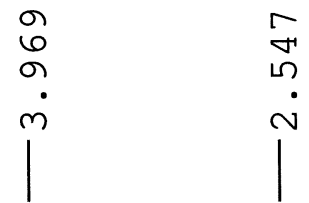

2

V

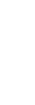

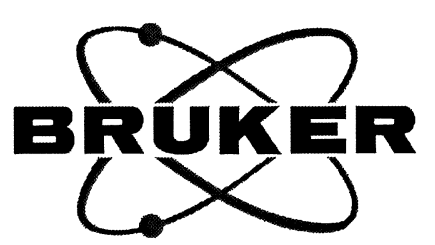

Current Data Parameters

NAME

EXPNO

PROCNO

F2 - Acquisition Parameters

Date 20201207

Time $\quad 10.33 \mathrm{~h}$

INSTRUM spect

PROBHD Z151340_0001

PROBHD Z151340_0001

$\begin{array}{lr}\text { PULPROG } & \text { zg30 } \\ \text { TD } & 65536\end{array}$

$\begin{array}{ll}\text { TD } & 65536 \\ \text { SOLVENT } & \text { CDCl3 }\end{array}$

SOLVENT

DS

SWH

FIDRES

AQ

DW

$\mathrm{DE}$

D1

TD 0

$\mathrm{SFO} 1$

NUC1

$\mathrm{PO}$

P 1

PLW1

$\mathrm{CDCl} 3$
16

$10504.202 \mathrm{~Hz}$

$0.320563 \mathrm{~Hz}$

$3.1195135 \mathrm{sec}$

47.600 usec

20.00 usec

$1.00000000 \mathrm{sec}$

$1.00000000 \mathrm{sec}$
1

$700.0115500 \mathrm{MHz}$

$1 \mathrm{H}$
.17 usec

9.50 usec

$13.00000000 \mathrm{~W}$

F2 - Processing parameters

SI 131072

SF $\quad 700.0070173 \mathrm{MHz}$

SFW

WDW

SSB

LB

PC

700.0070173
EM

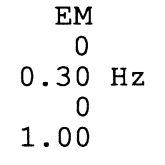




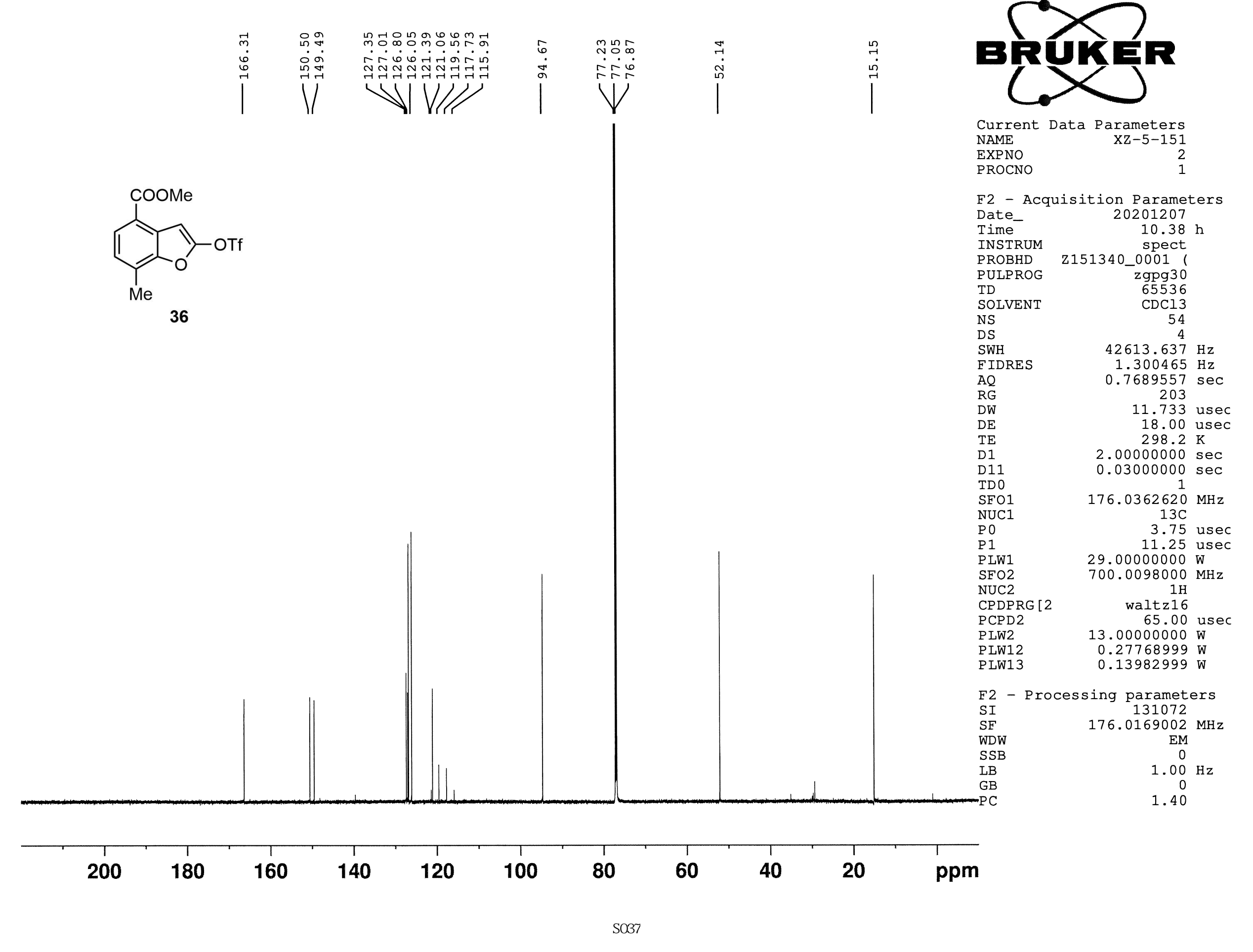


잉 ब $\infty \pi m \sim r$

$\therefore-\dot{r} \cdot \dot{2}$

- 111

37
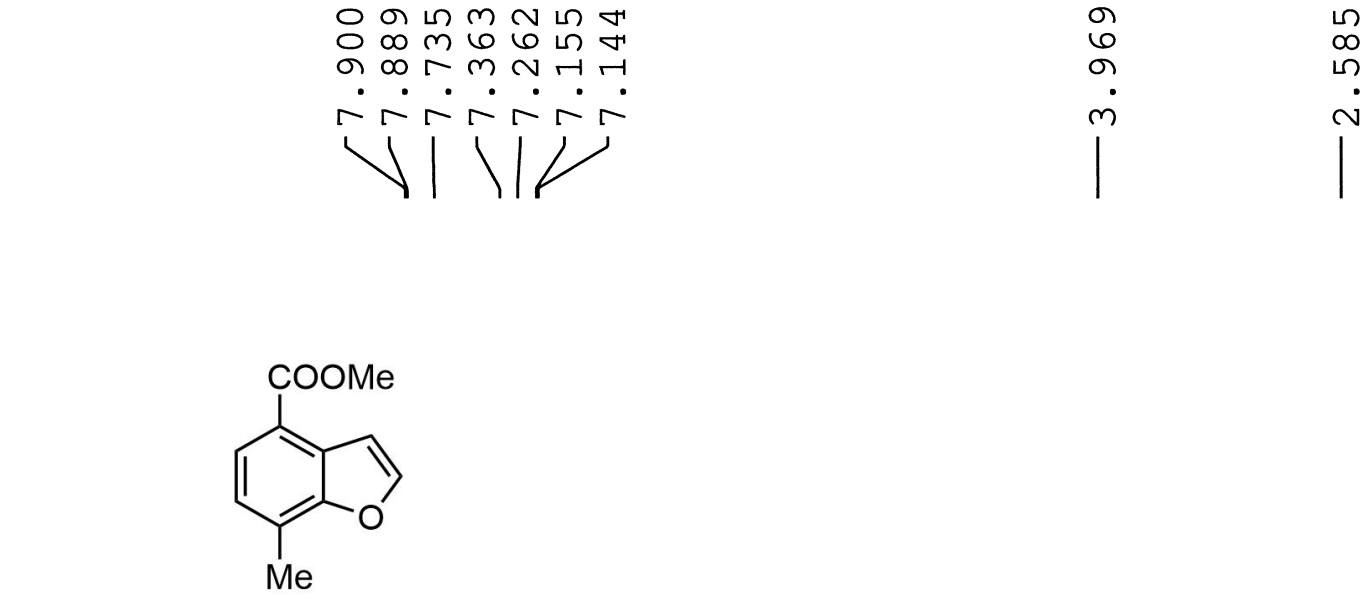

$\infty$

哽

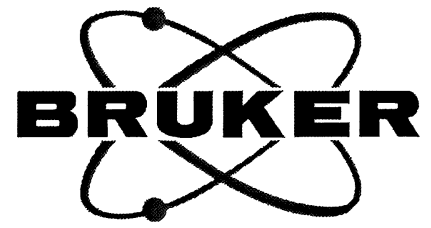

Current Data Parameters

NAME

EXPNO

PROCNO

F2 - Acquisition Parameters

$\begin{array}{lr}\text { Date_- } & 20201213 \\ \text { Time } & 16.52 \mathrm{~h}\end{array}$

$\begin{array}{ll}\text { Time } & 16.52 \\ \text { INSTRUM } & \text { spect }\end{array}$

PROBHD Z151340_0001)

PROBHD Z151340_0001

$\begin{array}{lr}\text { PULPROG } & \mathrm{zg} 30 \\ \text { TD } & 65536\end{array}$

SOLVENT CDCl3

NS

DS

SWH

FIDRES

AQ

RG

DW

DE

D1

TD 0

SFO1

NUC1

P0

P 1

16
2

$10504.202 \mathrm{~Hz}$

$3.1195135 \mathrm{sec}$

$$
\begin{array}{r}
36 \\
47.600
\end{array}
$$

47.600 usec

20.00 usec

$298.2 \mathrm{~K}$

$1.00000000 \mathrm{sec}$

700.0115500

$500 \mathrm{MHz}$

$1 \mathrm{H}$

3.17 usec

F2 - Processing parameters

SI 131072

SF $\quad 700.0070160 \mathrm{MHz}$

WDW

LSB

LB

GB

$\mathrm{EM}$
0

$0.30 \mathrm{~Hz}$

.00
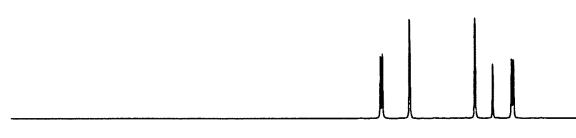

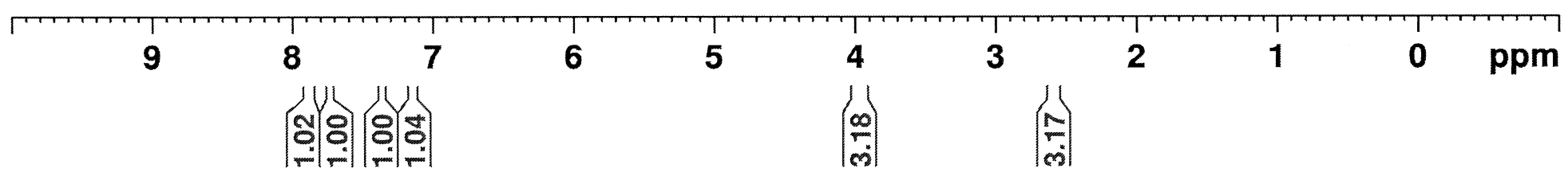




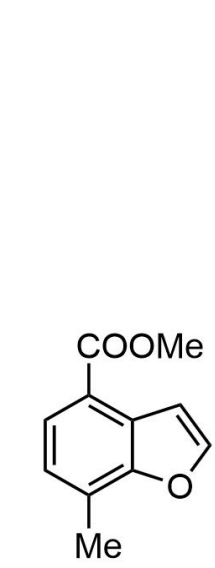

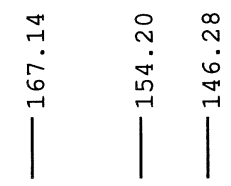

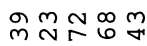

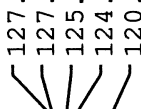

\begin{tabular}{l}
\multirow{0}{0}{} \\
0 \\
$\infty$ \\
0 \\
0
\end{tabular}

37

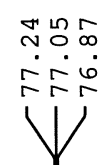

옥

ก$$
\text { ( }
$$ 


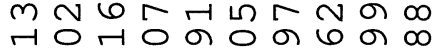

नु

- ivirior.i.

L

3

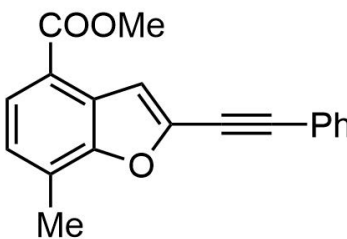

38

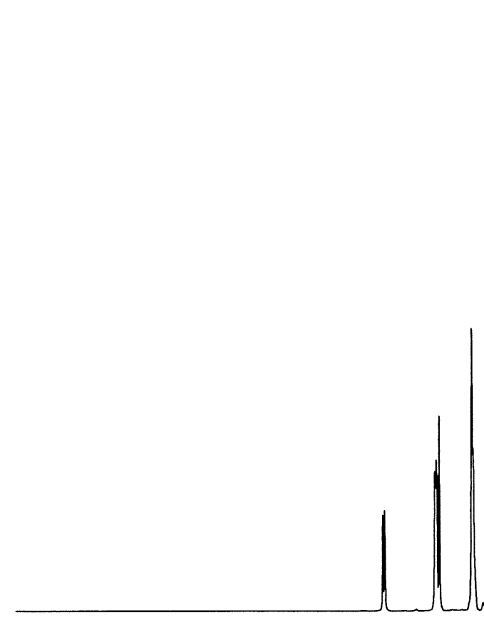

$\begin{array}{ll}\infty & \infty \\ \text { m. } & \text { के } \\ \dot{m} & \text { i }\end{array}$

の

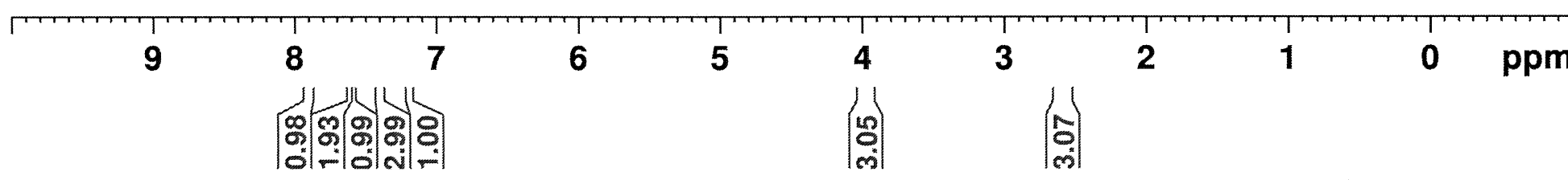




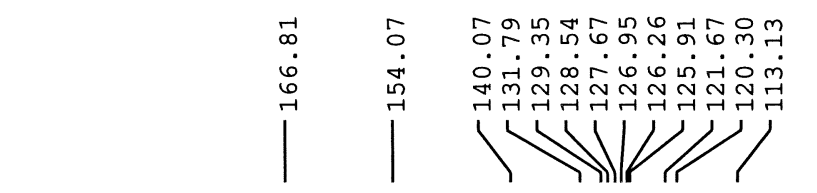

38
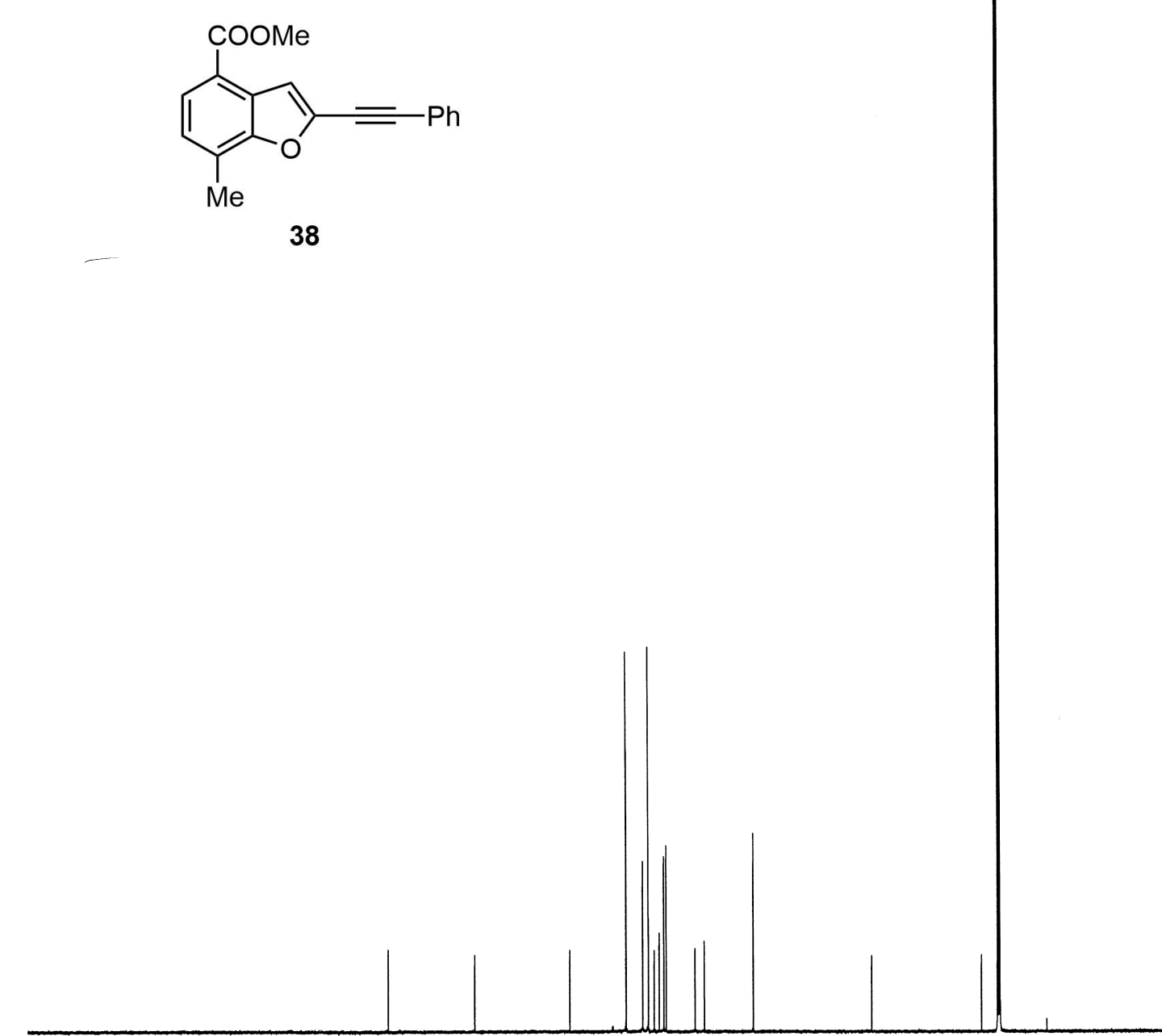

\section{Befren $C$}

Current Data Parameters NAME $\mathrm{Xz}-5-157$ EXPNO PROCNO

2

F2 - Acquisition Parameters Date__ 20201213

$\begin{array}{lr}\text { Date_ } & 20201213 \mathrm{~h} \\ \text { Time } & 16.46 \mathrm{~h}\end{array}$

INSIRUM spect

$\begin{array}{lr}\text { PROBHD } & \text { Z151340_0001 } \\ \text { PULPROG } & \text { zgpg30 }\end{array}$

$\begin{array}{lr}\text { PULPROG } & \text { zgpg30 } \\ \text { TD } & 65536\end{array}$

SOLVENT CDCl3

$\begin{array}{lr}\text { NS } & 25 \\ \text { DS } & 4\end{array}$

$\begin{array}{lr}\text { SWH } & 42613.637 \mathrm{~Hz}\end{array}$

$0.7689557 \mathrm{sec}$

AQ $\quad 0.7689557$

11.733 usec

18.00 usec $298.2 \mathrm{~K}$

$2.00000000 \mathrm{sec}$ $0.03000000 \mathrm{sec}$ $176.0362620 \mathrm{MHz}$ $13 \mathrm{C}$ 3.75 usec 11.25 usec $700.0098000 \mathrm{MHz}$

$$
1 \mathrm{H}
$$

65.00 use

$13.00000000 \mathrm{~W}$

$0.27768999 \mathrm{~W}$

$0.13982999 \mathrm{~W}$

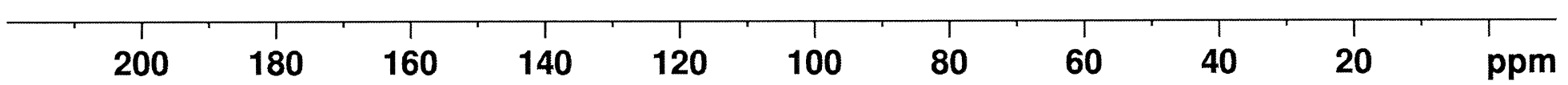


<smiles>CC(=O)c1ccc([N+](=O)[O-])c2oc(-c3ccccc3)cc12</smiles>

39

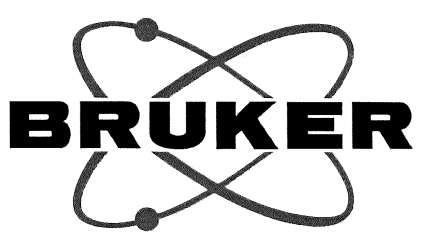

Current Data Parameters NAME TXPNO PROCNO $\mathrm{XZ}-5-164$ F2 - Acquisition Parameter

Time

INSTRUM

PROBHD

PULPROG

TD

SOLVENT

NS

DS

FIDRES

$F I D$
$A Q$
$R G$

RG
DW
DE

TE

D 1

$\mathrm{SFO} 1$

SEO1

NUC1

PLW1

Z151340_0001

z 930

65536

CDCl3 16

$10504.202 \mathrm{~Hz}$ $0.320563 \mathrm{~Hz}$ $3.1195135 \mathrm{sec}$ 36

47.600 usec 20.00 usec $298.2 \mathrm{~K}$

$1.00000000 \mathrm{sec}$

$700.0115500 \mathrm{MHz}$ $1 \mathrm{H}$

3.17 usec

9.50 usec

$13.00000000 \mathrm{~W}$

F2 - Processing parameters SI 131072

SF $\quad 700.0070177 \mathrm{MHz}$

WDW

SSB

LB

GB

0

EM

$0.30 \mathrm{~Hz}$

1.00

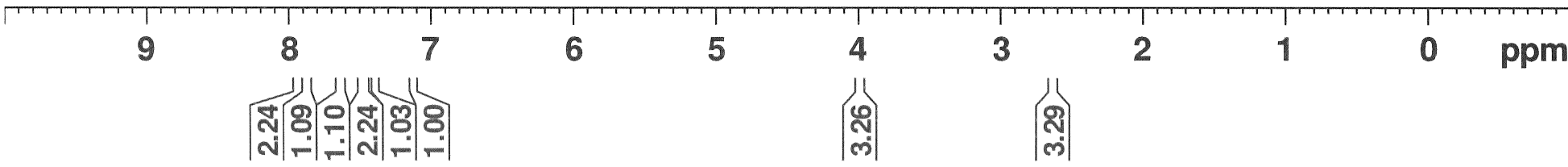




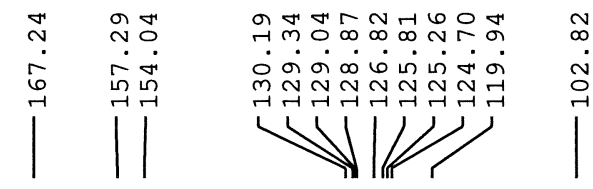

Me

39

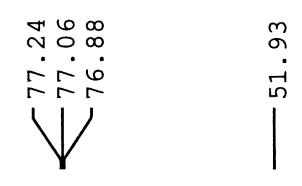

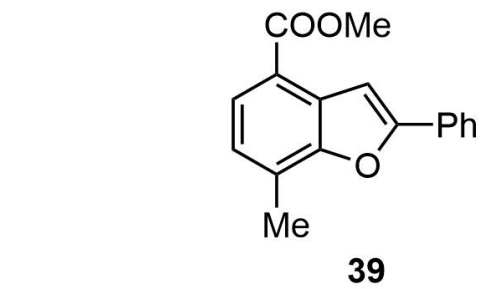

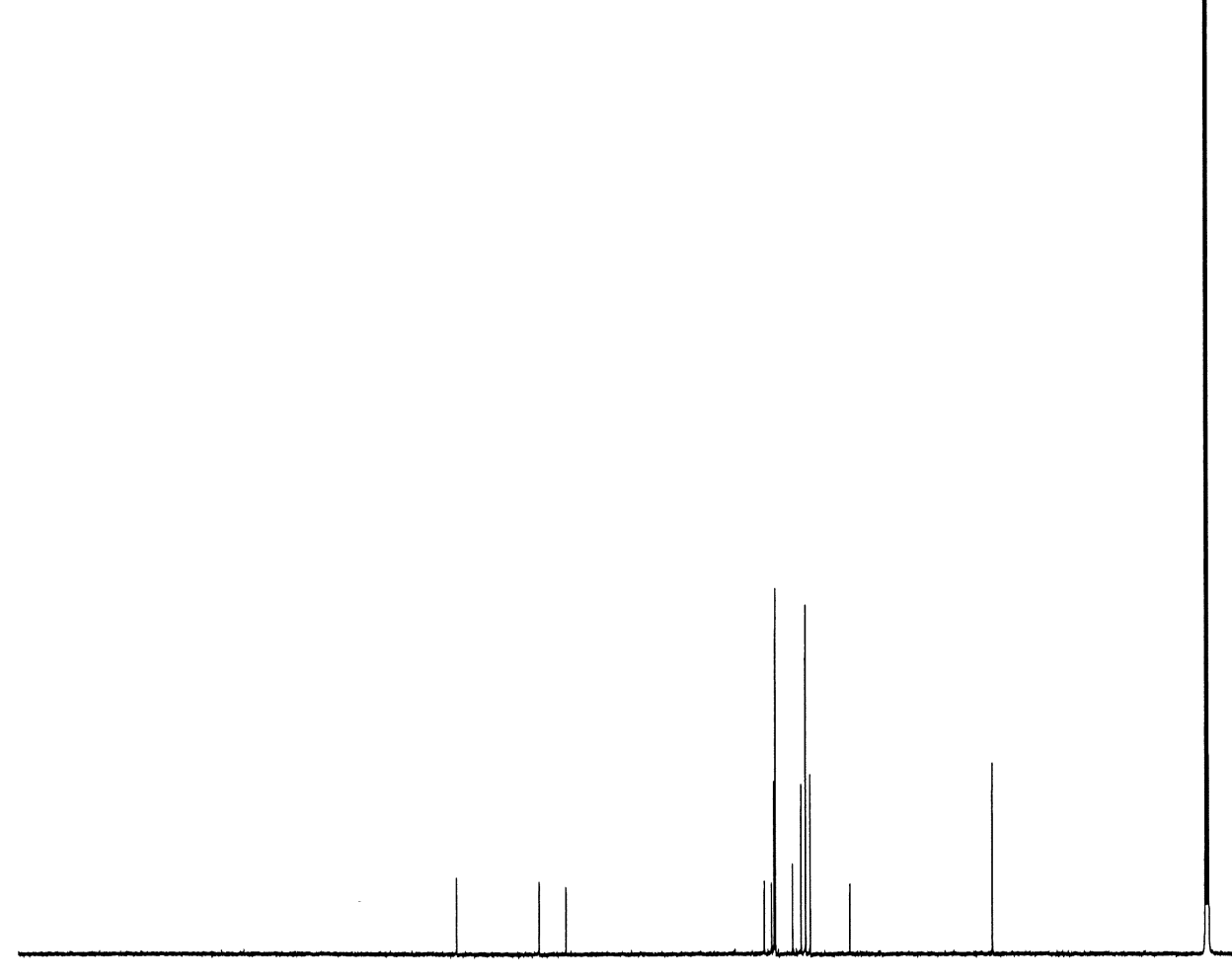

$=80$

Current Data Parameters

NAME

EXPNO

PROCNO

F2 - Acquisition Parameters

Date__ 20201221

Time $\quad 13.56 \mathrm{~h}$

INSTRUM spect

PROBHD Z151340_0001) (

PULPROG $\quad$ zgpg30

PDIP1

SOLVENT

NS

DS

SWH

FIDRES

$A Q$

RG

RG

$\mathrm{DE}$

$\mathrm{TE}$

D1 11

TD 0

$\mathrm{SFO} 1$

NUC1

$\mathrm{PO}$

$\mathrm{P} 1$

PLW1

$\mathrm{SFO} 2$

NUC2

CPDPRG [2

PCPD 2

PLW2

PLW12

PLW13

F2 - Processing parameters

SI 131072

SF $\quad 176.0169002 \mathrm{MHz}$

WDW

SS

LB

GB 